
\title{
3 Research Square \\ Deficient Uracil Base Excision Repair Leads to Persistent dUMP in HIV Proviruses During Infection of Monocytes and Macrophages
}

\section{Mesfin Meshesha}

Johns Hopkins University School of Medicine

Alexandre Esadze

Johns Hopkins University School of Medicine

Junru Cui

Johns Hopkins University School of Medicine

Natela Churgulia

Johns Hopkins University School of Medicine

\section{Sushil Kumar Sahu}

Johns Hopkins University School of Medicine

James Stivers ( $\boldsymbol{\nabla}$ jstiver1@jh.edu )

Johns Hopkins University School of Medicine https://orcid.org/0000-0003-2572-7807

\section{Research}

Keywords: Monocyte/Macrophage, HIV, dUMP, Uracil base excision repair

Posted Date: January 13th, 2020

DOl: https://doi.org/10.21203/rs.2.20758/v1

License: (9) (i) This work is licensed under a Creative Commons Attribution 4.0 International License.

Read Full License 


\section{Abstract}

\section{Background}

Non-dividing cells of the myeloid lineage such as monocytes and macrophages are target cells of HIV that have low dNTP pool concentrations and elevated levels of dUTP, which leads to frequent incorporation of dUMP opposite to A during reverse transcription ("uracilation"). One factor determining the fate of dUMP in proviral DNA is the host cell uracil base excision repair (UBER) system. Here we explore the relative UBER capacity of monocytes (MC) and monocyte-derived macrophages (MDM) and the fate of integrated uracilated viruses in both cell types to understand the implications of viral dUMP on HIV diversification and infectivity.

Results We find that monocytes are almost completely devoid of functional UBER, while macrophages are mainly deficient in the initial enzyme uracil DNA glycosylase (hUNG2). Accordingly, dUMP persists in viral DNA during the lifetime of a MC and can only be removed after differentiation of MC into MDM. Overexpression of human uracil DNA glycosylase in MDM prior to infection resulted in rapid removal of dUMP from HIV cDNA and near complete depletion of dUMP-containing viral copies. This finding establishes that the low hUNG2 expression level in these cells limits UBER but that hUNG2 is restrictive against uracilated viruses. In contrast, overexpression of hUNG2 after viral integration did not accelerate the excision of uracils, suggesting that they may poorly accessible in the context of chromatin. We found that viral DNA molecules with incorporated dUMP contained unique $(+)$ strand transversion mutations that were not observed when dUMP was absent $(G \rightarrow T, T \rightarrow A, T \rightarrow G, A \rightarrow C)$. These observations and other considerations suggest that dUMP introduces errors predominantly during (-) strand synthesis when the template is RNA. These mutations may arise from the increased mispairing and duplex destabilizing effects of dUMP relative to dTMP during reverse transcription. Overall, the likelihood of producing a functional virus from in vitro infection of $\mathrm{MC}$ is about 50 -fold and 300 -fold reduced as compared to MDM and activated $T$ cells.

Conclusions The results implicate viral dUMP incorporation in MC and MDM as a potential viral diversification and restriction pathway during human HIV infection.

\section{Background}

A role for the uracil base excision repair (UBER) pathway in HIV-1 infection of non-dividing macrophage and monocyte immune cells has been of interest for many years in at least two different biological contexts. These contexts arise from two innate immune responses that result in the introduction of dUMP into viral DNA [1-5]. These responses involve either enzymatic cytosine deamination of the viral $(-)$ strand cDNA by APOBEC DNA deaminases $[1,2]$ or the incorporation of dUMP opposite to adenine on either strand of viral DNA during reverse transcription $[3,4,6]$. The incorporation pathway arises specifically in quiescent cells because [dUTP] is typically higher in such non-dividing cells and most DNA polymerases readily utilize dUTP as a substrate in competition with dTTP [7-9]. The two uracilation 
pathways are quite unique because cytosine deamination leads to GàA transition mutations at specific trinucleotide sequences on (+) strand genomic RNA [10], while dUMP incorporation opposite to adenine occurs on both strands and is not intrinsically mutagenic [3]. Previous studies by us and others indicate that the majority of the dUMP that is present with in vitro infected monocyte-derived-macrophages (MDM) arises from dUMP incorporation by reverse transcriptase $[4,6]$. Once the viral DNA products enter the nuclear compartment, both types of lesions are substrates for uracil excision by the enzyme nuclear uracil DNA glycosylase (hUNG2)[3], the first enzyme in the UBER pathway. Excision by hUNG2 could lead to a variety of different outcomes ranging from viral DNA damage via strand breaks to replacement of dUMP with dTMP, restoring canonical T/A pairs $[4,6]$. We have previously reported that HIV DNA obtained from alveolar macrophages and circulating blood monocytes from drug naïve and ART patients both contained high levels of dUMP, while T cells from the same patients did not [4]. These findings suggest that uracilation occurs during in vivo HIV infection of both macrophages and their monocyte precursors.

It is not clear how HIV-1 remains infective in the hostile deoxynucleotide pool environment of quiescent cells, where overall dNTP concentrations are low and the ratio of [dUTP]/[TTP] is large $[11,12]$. One plausible explanation is that dUMP/A pairs are well-tolerated in viral CDNA and persist due to very low hUNG2 activity in non-dividing MDM [4]. The intrinsically low activity of hUNG2 in these cells could be further reduced by the ubiquitin-mediated degradation of hUNG2 facilitated by the HIV-1 accessory protein $\mathrm{Vpr}[4,13-15]$. In addition to promoting proteasomal degradation, Vpr has also been suggested to act as a transcriptional repressor of hUNG2 [16]. Another possible outcome in the context of low hUNG2 expression would be slow uracil excision, followed by repair. Over time, and after many excision/incorporation events, proviral dUMP would eventually be replaced with TMP, leading to canonical T/A base pairs. Support for such a replacement mechanism was previously suggested because the levels of dUMP in proviruses decreased over four weeks of culturing infected MDM [4]. The persistence of dUMP in viral DNA and the slow replacement mechanism have implications for viral latency in quiescent cells because in vitro and cellular transcription from uracilated DNA templates is greatly reduced even in the complete absence of hUNG activity [9]. This inhibitory effect of dUMP/A pairs arises at the level of transcription factor binding and RNA pol II activity $[9,17]$. The complexity of this biology that is unique to quiescent cells, challenges efforts to unravel the contributions of the component factors. Nevertheless, the fact that HIV-1 targets the UBER repair pathway at both the transcriptional and protein level indicates an evolutionary pressure to diminish the activity of this pathway during infection [16].

In this study we further explore the nucleotide profiles and UBER activities of MDM and their monocyte precursors with the aim of comparing the outcomes of dUMP incorporation and UBER activity during infection of these cell types (Fig. 1). The motivation for this work is the previous observation by us and others that HIV DNA is detected in circulating monocytes (MC) and alveolar macrophages (AM) in patients receiving ART who test negative for HIV RNA in blood samples (10-600 HIV copies have been detected per million MC or AM) $[4,18,19]$. Given that circulating MC are short-lived $\left(t_{1 / 2} \sim\right.$ several days) [20], the detection of HIV DNA in these cells of virus-free patients suggests that they arise from contact 
with latently infected cells in one or more HIV drug-resistant reservoirs, possibly by passing through a compartment that contains infected resident macrophages (or T cells) and then re-entering circulation. Productively infected MC could then seed infection in new tissues (Fig. 1)[21-23]. We now report that MC are much more deficient in UBER activities compared to MDM such that high levels of viral dUMP persist during the entire lifetime of a circulating MC. When infected MC eventually differentiate into MDM, the existing proviral dUMP can be slowly replaced by TMP using the UBER activities present in MDM. The implications for human HIV infection and potential therapeutic opportunities arising from these findings are discussed.

\section{Results}

dUTP/TTP in MDM and MC is high but variable with blood donors. In previous studies dUTP/dTTP ratios in the range $20: 1$ to $60: 1$ were reported $[4,12,24]$, while the ratio was essentially zero in dividing cells due to the expression of dUTPase in replicating cells [4]. These different estimates of intracellular dUTP/dTTP may arise from the combined challenges of measuring low levels of dNTPs present in nondividing cells, the inherent difficulty in accurately determining the ratio when one of the nucleotides is present at an extremely low level, intrinsic differences in dNTP pools in different donors, or differences in culture conditions or measurement methods.

We first extended the dTTP and dUTP measurements to freshly isolated monocytes with the expectation that the levels in freshly isolated cells would most closely match in vivo levels. A single nucleotide polymerase extension (SNE) assay was used where a 22 mer DNA primer-template containing a single adenine overhang on the template strand was extended by the dUTP or dTTP present in cell extracts (Fig. 2a)[3]. The assay takes advantage of the enzyme dUTPase to remove dUTP in the extract and allow measurement of dTTP in isolation (see methods). For these assessments, the [dTTP + dUTP] present in MC and MDM extracts were compared with the Hap1 dividing cell line (Fig. $\mathbf{2 b}$ and Supplemental Figure S1). We found similarly low levels of [dTTP + dUTP] in freshly isolated MC extracts as compared to MDM that had been differentiated over seven days in the presence of M-CSF (1.1 to $2.5 \mathrm{pmol} \mathrm{dU(T)TP/million}$ cells). For comparison, the combined [dTTP + dUTP] level in the Hap1 cell line is 25 to 70 -fold higher ( 70 pmol dU(T)TP/million cells). Depleting dUTP in these dNTP extracts by the addition of dUTPase allowed calculation of dUTP/dTTP in each cell type (Fig. 2c). Although freshly isolated MC and differentiated MDM showed similar elevated dUTP/dTTP ratios in the range 1:4 to 1:1 depending on the donor, the ratio was essentially zero for the HAP1 cells. Based on these and previous studies, we conclude that significantly elevated dUTP/dTTP is an intrinsic aspect of MC and MDM metabolism that differs from dividing cells.

Although a range of dUTP/dTTP values have been reported for MDM [4,24], all of the measurements are consistent with low dNTP pools and significant levels of dUTP for both MC and MDM. The current measurements indicate that uracilated viral CDNA will be produced during infection of both MC and MDM and that the UBER capacity of these cells could impact the outcome of infection. However, unlike previous studies where dUTP/dTTP $>20$, the more or less balanced ratio $(\sim 1)$ indicates that viral dUMP 
residues have the potential for being replaced by dTMP after multiple repair cycles (i.e. each replacement attempt has a 50:50 chance of replacing dUMP with dTMP, but eventually all dUMP would be repaired).

To evaluate whether HIV RTase can discriminate between dUTP and dTTP, and therefore bias dUMP incorporation away from the level expected from the dUTP/dTTP ratio, we measured the RTase activity in vitro using both dUTP and dTTP as substrates (Fig. 2d). Using a low dNTP concentration that approximated that calculated for non-dividing cells, we were unable to detect any selectivity of RTase for either nucleotide. Thus, the relative amounts of dUMP and dTMP in HIV reverse transcripts should reflect the cellular dUTP/dTTP.

MC and MDM have different uracil base excision repair (UBER) activities. Given that RTase shows no discrimination between dUTP and dTTP, the observed ratios of dUTP/dTTP of 0.25 to 1 for MDM and MC indicate that $99.9 \%$ of HIV DNA products would have at least one dUMP incorporation for every turn of the DNA helix assuming a random sequence containing 50\% A/T base pairs. These putative densely spaced uracils would be subject to excision by the UBER pathway. Accordingly, we were interested in the relative levels of seven enzymes involved in deoxyuridine metabolism in MC and MDM as compared to the Hap1 dividing cell line (Fig. 3). These enzymes included uracil DNA glycosylase (hUNG), AP endonuclease 1 (APE1), DNA polymerase $\beta$ (pol $\beta$ ), ligase Illa (the ligase isoform expressed in nondividing cells)[25], the dNTPase sterile alpha motif histidine-aspartate domain protein 1 (SAMHD1), and the DNA cytidine deaminases APOBEC3A (A3A) and APOBEC3G (A3G). The western blots and activity measurements for extracts collected from uninfected cells and MC and MDM that were infected with the CCR5 tropic HIV-1 ${ }^{\mathrm{BaL}}$ viral strain revealed the following general trends (Fig. 3a-3i and summary graphs in Fig. 3m-3s). First, MC and MDM show similarly low levels of expression of the first enzyme in the UBER pathway (hUNG), which are about 25 to 50-fold lower than the Hap1 reference line. These low hUNG activity levels, which could result from either the mitochondrial (hUNG1) or nuclear isoforms (UNG2), indicate that viral uracils may not be efficiently excised once viral DNA enters the nuclear compartment. Surprisingly, the activity of the next UBER enzyme, APE1, is highly expressed in both MDM and Hap1 cells, indicating that the rate-limiting step for repair of uracil in non-dividing cells is likely the initial excision event by hUNG2. The next two enzymes in the pathway, pol $\beta$ and lig IIla, are almost undetectable in MC but much more prevalent in MDM, although not to the same level as the Hap1 line. This important distinction between $\mathrm{MC}$ and MDM suggests that uracilated viruses generated by direct infection of $\mathrm{MC}$ cannot be repaired until the MC differentiate into MDM. As expected, SAMHD1 dNTPase is highly expressed in both MC and MDM, but not Hap1 cells, consistent with the different dNTP pool levels present in these cell types (Fig. 2b). Both deaminase enzymes, A3G and A3A, are highly expressed in MC, but not MDM or Hap1 cells. Despite the high expression levels of $A 3 G$ and A3A, the high dUMP content of viral DNA in MC is not derived from intrinsic $A 3 A$ or $A 3 G$ activity-at least not with $\mathrm{HIV}-1^{\mathrm{Ba}}{ }^{\mathrm{v}}$ virus that encodes viral infectivity factor ( $v i f$ )(see below). None of the expression data were noticeably affected by whether the extracts were prepared before or after BaL virus infection (Fig. 3). We performed additional quantitative RT-qPCR measurements of mRNA expression levels of these genes which were fully consistent with the above trends (Supplemental Figure S2). 
HIV Infection of MC and MDM at early and late stages of differentiation. To explore the origins and possible fate of infected circulating monocytes observed in HIV patients on ART, we performed HIV infections using freshly isolated, undifferentiated MC rather than fully differentiated MDM. We began this exploration by measuring the levels of dUMP present in proviral DNA under three distinct conditions (i) $\mathrm{MC}$ that were infected with HIV-1 ${ }^{\mathrm{BaL}}$ immediately after isolation and then maintained as undifferentiated monocytes for 7 days by culturing under nonadherent conditions in the absence of M-CSF, (ii) MDM infected at the monocyte stage and then allowed to differentiate in the presence of M-CSF (Early Infection, $\mathrm{MDM}^{\mathrm{EI}}$ ) and, (iii) MDM that were infected after seven days of differentiation in M-CSF (Late mfection, $\mathrm{MDM}^{\mathrm{L}}$ ) (Fig. 4a). For comparison, we infected activated T cells using the same virus stock and MOI. Proviral dUMP levels were determined using the alu-gag Ex-qPCR experiment after isolating genomic DNA at 1, 3, 7, 14- and 28-days post infection (dpi). To prevent multiple round infection, $10 \mu \mathrm{M}$ enfuvirtide (T-20) was added one day after the initial infection (Fig. 4a). The Ex-qPCR analysis determines the fraction of integrated HIV viruses in a DNA sample that contain one or more dUMP residues on each DNA strand of a DNA amplicon that contains a 650 bp region of the $5^{\prime}$ LTR and a 700 bp portion of the gag gene $[4,26]$. For MC, which were cultured using non-adherent conditions in the absence of M-CSF to maintain a monocyte-like phenotype (Supplemental Fig. S3), no proviral DNA was detected at $1 \mathrm{dpi}$, but by day three nearly $100 \%$ of the proviral copies contained dUMP, which persisted until the end of the experiment (7 dpi)(Fig. 4b). Due to limitations in the length of time MC can be maintained using nonadherent conditions, this experiment could not be continued beyond seven days. For MDM ${ }^{\mathrm{EI}}$, which were infected immediately after isolation of $\mathrm{MC}$ and then cultured using adherent conditions in the presence of M-CSF to immediately begin their differentiation into MDM, over $50 \%$ of the provirus contained dUMP at $1 \mathrm{dpi}$, which increased to almost $100 \%$ at $3 \mathrm{dpi}$. Unlike MC grown under non-adherent conditions in the absence of M-CSF, the copies of HIV in MDM ${ }^{E l}$ that contained dUMP decreased by 7 dpi (Frac U 75\%) and even further at $14 \mathrm{dpi}$ (Frac U 20\%). For $\mathrm{MDM}^{\mathrm{LI}}$, which were infected after complete differentiation, the proviral copies that contained dUMP were initially lower than MC or MDM ${ }^{\mathrm{EI}}$ (Frac U $15 \%$ at $1 \mathrm{dpi}$ and $60 \%$ at $7 \mathrm{dpi}$ ), but ended up at the same level as $\mathrm{MDM}^{\mathrm{El}}$ at $14 \mathrm{dpi}$ (Frac $\mathrm{U} \sim 20 \%$ ). We attribute the lower Frac $U$ values of differentiated MDM at early times after infection to the greater rate of reverse transcription in a small sub-population of permissive MDM $(<10 \%)$ which have higher dNTP pools and low dUTP [4]. As the post-infection time increases, the slower replicating uracilated DNA products in the major MDM population increasingly contributes to the bulk measurement. The control T cells showed no viral-associated dUMP at any time during infection, consistent with our previous finding (4).

We then investigated the kinetics for appearance of early and late reverse transcripts (ERT, LRT) and proviral DNA using the three infection conditions. The kinetics for forming ERT and LRT products followed the trend $\mathrm{MDM}^{\mathrm{LI}}>\mathrm{MDM}^{\mathrm{El}}>\mathrm{MC}$ (Fig. 4c, d), with ERT and LRT copy numbers for MDM ${ }^{\mathrm{El}}$ and MC about 2 and 6 -fold lower at $7 \mathrm{dpi}$ as compared to $\mathrm{MDM}^{\mathrm{LI}}$. A similar trend was observed for the proviral copy numbers (Fig. 4e). The control infections of activated T cells showed both faster reverse transcription kinetics ( $~ 70 \%$ complete in $\sim 1$ day, Fig. $4 \mathbf{c}, \mathbf{d}, \mathbf{e})$ and a greater number of integrated proviruses. 
dUMP in proviral DNA does not arise from cytidine deaminase activity in MC. Given the presence of APOBEC enzymes in MC, and to a lesser extent MDM as judged by immunoblotting (Fig. 3a, g), we wanted to confirm that most of the dUMP in HIV proviral DNA was derived from incorporation of dUMP by RTase. One established way to test this is to add high levels of thymidine (dThyd) to the cell culture media prior to infection to increase the levels of intracellular TTP and then look for a reduction in the number of viral DNA products that contain dUMP [4]. We infected both MC and fully differentiated MDM in the presence of $5 \mathrm{mM}$ dThyd and measured the copies of uracilated proviruses at $7 \mathrm{dpi}$ using the Alugag Ex-qPCR method (Fig. 5). For both cell types we observed a 4-8-fold reduction in uracilated viral copies, indicating a predominant role for dUMP incorporation. A further reduction in uracilated viral copies is not expected due to the minor population of highly permissive MDM that do not contain high dUTP levels [4,27]. A minor role for APOBEC DNA cytidine deamination is confirmed by our proviral DNA sequencing results reported below, which show a low frequency of GàA transition mutations on the proviral (+) strand DNA at known APOBEC hotspots.

Effect of viral dUMP on proviral DNA and extracellular RNA sequences. Although dUMP incorporation is not expected to introduce mutations because its Watson-Crick hydrogen bond donor and acceptor groups are identical with thymidine, dUMP/A base pairs have reduced thermodynamic stability in the context of B DNA [28], increased base pair dynamics [29], and dUMP has an increased propensity to form dUMP/G wobble mismatches relative to thymine due to its larger keto-enol tautomerization constant. The reduction in duplex stability arising from dUMP has been attributed to the lower electron density of the pyrimidine ring system of uracil relative to thymidine, which weakens base stacking [29]. Thus, we were interested if any of these potential effects of dUMP incorporation could be detected in CDNA sequences produced from reverse transcriptase or viral RNA genomes produced by RNA pol II transcription.

To investigate proviral DNA sequences produced by reverse transcription in both MC and fully differentiated MDM, we used HIV-1 ${ }^{\mathrm{BaL}}$ virus to infect freshly isolated MC cultured under non-adherent conditions and also MDM after 7 days of culturing. Both cell types were cultured for seven days after infection and total DNA was isolated and diluted to the single copy level. Single

proviral clones were then amplified using ES7 and ES8 env primers (see Supplemental Table S1). Wells that were positive for HIV DNA clones by qPCR were reamplified using primers that generated a $592 \mathrm{bp}$ amplicon covering the V3 and V4 regions of env, followed by sequencing using the Sanger method. The viral sequences were compared with the lab reference sequence to determine the mutation frequencies and types. For proviral sequences isolated from MC and MDM, the viral mutation frequency $\left(\sim 1.5 \times 10^{-3}\right)$ and mutational spectrum were similar (Table 1, Fig. 6a, b). For both cell types, 26 to $38 \%$ of the isolated proviral clones contained substitution mutations, with the majority appearing as transition mutations ( 70-80\%), and the remaining being more unusual transversion mutations (20\% to $30 \%)$. Two (+) strand GàA mutations detected in both MDM and MC infections might be attributed to enzymatic cytosine deamination on the viral (-) strand cDNA based on the sequence preferences of A3A or A3G (Supplementary Table S2). However, for MDM we cannot exclude that these apparent enzyme derived mutations arose from chance misincorporation because four GàA mutations occurred on the viral (-) 
strand cDNA (corresponding to CàT on the positive strand). These mutations in MDM cannot be attributed to APOBEC activity because of their sequence context and occurrence on the (-) strand (Supplementary Table S2). The two (+) strand GàA mutations observed in the MC derived samples could arise from the high A3A activity in these cells. Finally, $40 \%$ and $55 \%$ of the proviral mutations in MDM and MC led to codon changes and could therefore affect viral fitness (Supplementary Table S2, S3).

Since the mutational spectrum for integrated virus was indistinguishable for $M C$ and MDM, we chose to selectively sequence extracellular viral RNA produced from HIV-1 ${ }^{\mathrm{BaL}}$-infected MDM (Fig. 6c). The viral RNA sequences showed a slightly elevated mutation frequency compared the proviral DNA $\left(1.7 \times 10^{-3}\right)$ and similar percentages of transition and transversion mutations. In addition, about $65 \%$ of the viral RNA mutations led to codon changes (Supplementary Table S5). We recently reported a mutational frequency of $0.6 \times 10^{-3}$ for RNA pol II transcription using linear uracilated DNA templates that were transfected into human UBER deficient Hap1 cells [9]. In this study, the 2.3-fold higher mutation frequency arising from reverse transcription obscures any additional viral RNA mutations that might arise from RNA pol II transcription.

Selective sequencing of dUMP-depleted DNA fraction. We were interested in whether a different mutational spectrum might result if the fraction of proviral DNA that contained dUMP was subtracted from the total population of proviral DNA before single-molecule amplification and sequencing. To address this question, we performed UNG digestion on the total DNA extracted from infected MDM to remove all of the dUMP-containing copies prior to PCR amplification and then repeated the limiting dilution steps and clonal sequencing (Fig. 7a). Although the dUMP-depleted DNA showed a modest 2fold reduction in the mutation frequency $\left(0.8 \times 10^{-3}\right)$ (Table 1, Supplemental Table S4), this reduction was not highly significant ( $p$-value $=0.16$ ). Despite the insignificant effects on the mutation frequency, the mutation spectrum for the dUMP-subtracted DNA fraction consisted almost entirely of transition mutations, with only a single transversion mutation after sequencing 22,000 bases (Fig. 7a). We calculate that there is only a $1 \%$ probability that a single transversion mutation would have been randomly observed in the dUMP-depleted sample based on Poisson statistics and the measured transversion frequency in the dUMP-rich DNA sample. Thus, transversion mutations appear to be correlated with the DNA fraction that contains dUMP.

To explore why DNA clones containing dUMP showed a higher frequency of transversion mutations, we examined the average $[A+T(U)]$ content for 7 mer sequences centered on these transversion sites to explore whether the mutations might be correlated with the density of dUMP incorporation on either the $(+)$ or $(-)$ strand. For comparison, we determined the average $[A+T(U)]$ content of the 7 mer sequences surrounding the transition mutation sites in the dUMP-depleted and total DNA samples (Supplemental Tables S2 and S4). This comparison showed a statistically higher average frequency of $[A+T(U)]$ near the transversion sites $(\mu=0.80)$ as compared to the transition mutation sites (dUMP-depleted DNA, $\mu=$ 0.58 ; total DNA, $\mu=0.55$ ) (Fig. $7 b$ ). The differences in the mean values are significant with $p$ values < 0.003. Possible mechanistic implications of this result are discussed below. 
Relative infectivity of $\mathrm{HIV}-1^{\mathrm{BaL}}$ produced from $\mathrm{MC}, \mathrm{MDM}^{\mathrm{El}}$ and $\mathrm{MDM}^{\mathrm{L}}$. We determined the relative efficacy by which MC, MDM and activated T cells produce virus particles and extracellular vRNA by normalizing the viral output by the average number of proviruses present in each infected cell type (Fig. 8a, b). Using p24 or extracellular viral RNA levels as the measure for output, there were only modest differences for the three myeloid cell infections at $7 \mathrm{dpi}$, although MDM that were infected after complete differentiation $\left(M^{2} M^{L I}\right)$ showed a 2-fold greater yield than $M C$ or $M^{2} M^{E I}$. For comparison, activated T cells infected under identical conditions showed a 5 -fold higher viral output than MC (Fig. 8a, 8b), indicating an intrinsically higher efficiency than any of the infected myeloid cells.

Finally, we investigated the relative infectivity of $\mathrm{HIV}-1^{\mathrm{BaL}}$ produced from infected $\mathrm{MC}, \mathrm{MDM}^{\mathrm{EI}}, \mathrm{MDM}^{\mathrm{LI}}$ and activated T cells by collecting culture supernatants at $7 \mathrm{dpi}$ and using equivalent copies of virus to infect MOLT-4/CCR5 target cells (Fig. 9a). Seven days after infection of the target cells, total cellular DNA was extracted, and LRT copies were quantified using QPCR (Fig. 9b) and p24 levels were measured in the culture media (Fig. 9c). Although equal p24 units were used in each of the infections, the target cell LRT copies were about 4-fold greater for infections initiated with viral supernatants obtained from $\mathrm{MDM}^{\mathrm{LI}}$ as compared to $\mathrm{MC}$, while $\mathrm{MDM}^{\mathrm{El}}$ showed an intermediate level of LRT copies. In addition, supernatant p24 levels were 6-fold greater for infections initiated with $M M^{L}$. These differences indicate that the fitness of viral particles produced from MC is lower than MDM despite the similar efficiency of production from integrated viruses (Fig. 8). If we define an infection cycle that begins with viral infection of a MC or MDM and ends with the infection of a new target cell, we calculate an overall 50-fold lower efficiency for MC as compared to a fully differentiated MDM. This comparison between the two cell types is based on the product of the relative efficiencies for virus integration (Fig. 4), viral particle production from integrated virus (Fig. 8) and the productive infection of new target cells (Fig. 9). Using the same calculation, MC are 300 -fold less efficient at producing infective virus as compared to activated $T$ cells. It is not clear at this time how much viral dUMP levels or UBER contributes to the lower infection efficiency in myeloid cells.

Expression of exogenous hUNG2 before HIV infection depletes uracilated HIV DNA products. Because of the vanishingly low levels of UBER pathway enzymes detected in MDMs, and especially the very low hUNG2 activity observed in both monocytes and MDMs, we hypothesized that low hUNG2 levels might limit the restrictive effects of the UBER pathway. To test this hypothesis, we over expressed full length human UNG (hUNG2) in MDMs using a doxycycline inducible lentiviral transduction system. The inducible system allowed us to test whether hUNG2 expression had a greater effect before HIV infection, or alternatively, after HIV had integrated into the MDM genomic DNA. Control experiments demonstrated the presence of high hUNG2 activity in cell extracts prepared from transduced MDM at days 1 and 3 post induction, but no activity in the absence of doxycycline induction (Supplemental Fig. S5).

We first tested the effect of inducing hUNG2 expression prior to infection with VSVG pseudo-typed HIVPNL4-3 virus particles capable of only a single round infection. In this experiment, fully differentiated MDM were first transduced with the hUNG2 expressing lentivirus at an $\mathrm{MOI}$ of 5 ( $0.1 \mathrm{pg} \mathrm{p24}$ antigen/cell) and expression of hUNG was induced using $1 \mu \mathrm{g} / \mathrm{ml}$ of doxycycline three days after transduction, 
followed by infection with HIVNL4-3 one day later (MOI = 0.5) (Fig. 10a). We followed the HIV provirus copy number and the fraction of total provirus that contained dUMP using alu-gag Ex-qPCR. These measured outcomes were compared with those of an uninduced control infection (blue bars, Fig. 10a), as well as an infection with HIVPNL4-3 in the absence of any prior lentiviral transduction (green bars, Fig. 10a). With preinfection induction of hUNG2 expression (red bars, Fig. 10a), there was a 50\% decrease in the provirus copy number between 1- and 7-days post HIVpNL4-3 infection and the fraction of proviruses containing dUMP was at the limit of detection for the Ex-qPCR method $(<0.2)$. In contrast, the no induction and $\mathrm{HIV}^{N L 4-3}$ only control infections showed a stable or slightly increasing proviral copy number over the same time period and the fraction of proviruses containing dUMP increased from $\sim 0.4$ to 0.8 . As discussed above and previously[4,27], no more than a $50 \%$ loss of provirus is expected when hUNG 2 is overexpressed because the MDM exist as a mixed population where only about $40-50 \%$ of the total proviruses contain dUMP. However, the essentially complete absence of viral dUMP at one to seven days post infection as measured by alu-gag Ex-qPCR clearly indicates cellular hUNG2 excised the uracils prior to viral integration. These results indicate that when hUNG2 is abundant in the target cell prior to HIV infection, uracilated viruses can be efficiently destroyed before integration.

In a second experimental protocol, hUNG2 induction was delayed until 7 days after HIVNL4-3 infection to allow most of the HIV cDNA to integrate (Fig. 10b). Under this infection scenario we saw a smaller decrease in HIV proviral DNA between 8- and 14-days post-infection (one to seven days post-induction) as compared to the pre-infection induction of hUNG2 shown in Figure 10a. In addition, a higher fraction of proviruses contained dUMP and these proviruses slowly disappeared over one to seven days postinduction. In fact, the rate of decrease of dUMP-containing proviruses was not significantly different for the hUNG2-induced condition and the uninduced controls (Fig. 10b). This indicates that the rate-limiting step for post-integration excision of uracils does not involve hUNG2 and may instead involve remodeling of chromatin.

\section{Discussion}

dUTP-mediated innate immunity was first suggested for $\beta$-retroviruses, non-primate lentiviruses, and endogenous retroviruses [30-33]. These viruses have all captured a host dUTPase gene during viral evolution, which is a powerful enzyme that degrades dUTP to dUMP and $\mathrm{PP}_{\mathrm{i}}$, promoting infection of macrophages by these viruses. Although HIV-1 does not encode for dUTPase, it can infect human monocytes and macrophages even though the ratio dUTP/TTP is large. One strategy that HIV uses to replicate in this environment is to code for the accessory protein vpr, which is used to target hUNG2 for ubiquitination and proteasomal degradation [16,34], suggesting that incorporation of dUTP is tolerated but that the antiviral function of hUNG2-catalyzed uracil excision must be overcome. Consistent with this phylogenetic comparison of different retroviruses, our previous work established that this dUTP-mediated restriction pathway works most potently when target cell dUTPase expression is low, dUTP is abundant, and nuclear hUNG2 is highly expressed so as to efficiently degrade viral CDNA [3,4]. When any of these elements are absent or diminished, the effectiveness of the restriction pathway is diminished, which likely 
explains diverse reports in the literature concerning the impact of this pathway [31, 35-37]. Accordingly, quantification of cellular [dUTP]/[dTTP] and UBER enzyme expression levels are key to understanding the impact of this pathway in MC and MDM.

Implications of UBER activity and [dUTP]/[dTTP] in MC and MDM. Since MC differentiate into macrophages, it is possible that a macrophage originally infected at the MC stage of differentiation might produce different amounts and sequences of virus compared to a macrophage that was infected after complete differentiation (Fig. 1). This question pertaining to the order of infection is relevant because MC containing uracilated viral DNA have been detected in blood samples of HIV patients on ART who are in full remission (10-600 infected $\mathrm{MC} / 10^{6}$ cells) $[4,19]$. This observation is intriguing because peripheral blood $\mathrm{MC}$ have a limited half-life of about 2 days in circulation and in this limited period infection must occur. After infection, MC either migrate to lymphoid tissues and differentiate into macrophages or are eliminated [20]. We speculate that under virally repressed conditions MC are most likely infected while passing through a tissue reservoir containing HIV infected cells and then enter back into circulation via retrograde migration before dissemination to other tissues $[22,23]$.

Here we have demonstrated the kinetic competence of the MC infection pathway in vitro through the detection of viral cDNA products in MC within a relevant time frame (Fig. 4c-e). Since MC have high dUTP levels, but the entire UBER pathway is almost absent with the exception of APE1 (Fig. 3), dUMPcontaining proviruses likely persist throughout the $\mathrm{MC}$ lifetime. Thus, infected $\mathrm{MC}$ harboring uracilated viral DNA may successfully migrate to a tissue compartment and differentiate into macrophages. Once differentiated into macrophages, UBER activity is upregulated and dUMP can be slowly excised and at least partially replaced with dTMP. This mechanism is supported by the observed slow disappearance of viral dUMP over 28 days in MDM, with no change in proviral copy number, suggesting that dUMP is being replaced by dTMP via UBER (Fig. 4b). This mechanism requires that the dTTP concentration is competitive with dUTP to prevent futile cycling (futile cycling is the excision of dUMP and its reincorporation by DNA pol). The measured [dUTP]/[dTTP] $\sim 1$ in macrophages in our in vitro culture conditions is compatible with a repair mechanism that allows slow nucleotide replacement over time (Fig. 2). In contrast, the [dUTP]/[dTTP] in the range 20 to 60 as found in two previous studies would effectively prevent repair, and excision of such densely spaced uracils by hUNG2 would likely lead to fragmentation of the viral DNA as previously indicated $[3,4,24]$. If the [dUTP]/[dTTP] ratio is variable in vivo, it will have an impact on the efficiency of HIV infection of myeloid lineage cells, with outcomes ranging from slow repair to viral destruction depending on the dUMP density and hUNG2 expression levels.

UBER may occur after switching of macrophages from a $\mathrm{G}_{0}$ to $\mathrm{G}_{1}$ cell cycle state. Mlcochova et al reported that non-dividing MDM exist in two interconvertible cell cycle populations, a minor population that exhibits markers of $G_{1}$ and is DNA repair competent and a predominant population that is $\mathrm{G}_{0}$-like and deficient in the expression of many DNA repair proteins and DNA replication components [27]. The two states can also be detected by infection with an HIV construct containing an eGFP expression cassette [4, $27,38]$. In this case, the restrictive $G_{0}$ population shows little or no eGFP fluorescence, even though viral 
cDNA is present in nearly every cell, while the permissive G1 population shows high eGFP expression [4, 38]. These observations of Mlcochova et al parallel our previous measurements using a fluorescent reporter virus [4]. In our previous study we sorted infected MDM into GFP positive and negative populations and found that the GFP positive $\left(\mathrm{G}_{1}\right)$ population did not contain any detectable dUTP and the viral DNA was free of dUMP (4). In contrast, the GFP-negative $\left(\mathrm{G}_{0}\right)$ population contained high [dUTP] and the viral DNA also contained high levels of dUMP [4]. More recent work on the effects of dUMP on transcription of DNA templates by RNA pol II establishes that the differences in GFP expression between the two populations is at least partly due to transcriptional repression by dUMP [9]. Thus, these combined findings suggest that UBER may occur after an infected $G_{0}$ macrophage transiently moves to the $G_{1}$ state where the nucleotide pool composition and DNA repair capacity can facilitate dUMP removal and replacement. We speculate that small molecules that target the $G_{0}-G_{1}$ checkpoint might be useful to induce the transition to the $G_{1}$ state and potentially lead to inactivation of heavily uracilated proviruses by upregulation of UBER.

The realization that bulk MDM consist of two cell populations with respect to dUTP levels and UBER capacity further informs our understanding of the observation that only a fraction of total viral cDNA molecules contain dUMP. For instance, assuming a homogenous MDM population, it is difficult to rationalize why only $\sim 60 \%$ of total proviruses contain dUMP within a 1200 base pair amplicon (Fig. 4b). This fraction is enigmatic because with [dUTP]/[dTTP] $~ 1$ and the inability of reverse transcriptase to discriminate between dTTP and dUTP (Fig. 2), every 10 bp of viral DNA should contain dUMP on both strands and all proviruses should contain dUMP. Thus, the low fraction of uracilated proviruses in MDM is consistent with the presence of two cell sub-populations and only one of the populations $\left(G_{0}\right)$ contains high [dUTP]/[TTP]. In contrast, the observation that nearly all copies of HIV contain persistent dUMP during infection of $M C$ suggests that the $G_{1}$ population does not exist to a significant extent before differentiation into MDM. Consistent with this model, MC show virtually no eGFP expression upon infection with a GFP reporter virus even though proviral DNA is present in nearly every cell (Supplemental Figure S4).

Potential mechanisms for mutational effects of dUTP incorporation by reverse transcriptase. There are surprisingly few studies of the mutational events during HIV infection of MDM, and to our knowledge, no previous reports of HIV sequences derived from infection of MC. The most complete and direct comparison of HIV mutations arising during single round infection of T cells and MDM is the nextgeneration sequencing study of full-length HIV genomes by Cromer et al [39]. In this study, the reported overall mutation frequency was $\sim 1 \times 10^{-4}$ for both cell types. Their reported HIV mutation spectrum for MDM infections $(G \diamond A=35 \%, A \diamond G=18 \%, A \diamond T=17 \%, A \diamond C=11 \%)$ does not differ significantly from the profile obtained in our selective sequencing of a $500 \mathrm{bp}$ amplicon covering the $\mathrm{V} 3$ and $\mathrm{V} 4$ regions of env, which is known to be highly variable (Fig. 6b) [40, 41]. However, our mutation frequency for this select region $\left(1.4 \times 10^{-3}\right.$, Table 1$)$ is significantly higher than the average value previously reported by Cromer et al for the entire HIV sequence. This result is not unanticipated because we have previously reported that 
the mutation frequency for HIV DNA and RNA isolated from infected MDM is variable across the HIV genome [4]. In particular, the LTR region has very low levels of mutations [4].

One significant observation in our sequencing studies was that transversion mutations in proviral DNA were not detected in DNA samples where the dUMP fraction was subtracted by UNG digestion (Table 1, Fig. 6c). This result indicates that the mutational profile for misincorporation of nucleotides by reverse transcriptase is indirectly impacted by the presence of dUMP in the (-) or (+) strand sequences. Although the detailed mechanistic basis for this outcome is not known, the sites of these transversion mutations are especially AT(U) rich (Fig. 7b), suggesting that uracils on either strand may be involved (Supplemental Table S2). There is currently no information about the stability or dynamics of dUMP base pairs in the context of RNA/DNA hybrids, the possible sequence contexts that might give rise to mutational hotspots, or the activity of reverse transcriptase under such conditions. Relevant to the current findings, previous work on dUMP/A base pairs in duplex DNA has shown increased base pair dynamics and decreased thermodynamic stability $[28,29,42]$. The increased dynamics has been studied using NMR imino proton exchange and attributed to the reduced electron density of the base, which leads to poorer stacking interactions with neighboring bases [29]. Such effects in the context of RNA/DNA hybrids containing densely spaced dUMP substitutions are anticipated but require detailed biochemical studies to confirm. In this regard, we recently reported reduced fidelity of T7 RNA polymerase during transcription of RNA using a DNA template that contained dUMP [9]. These mutations were also explained by indirect effects of dUMP arising from destabilization of the template-primer, which increased strand slippage and realignment errors during polymerization. Similar observations with reverse transcription now suggest this proposed effect of dUMP may be general.

A further aspect of interpreting the mutational spectrum that arises during dUMP incorporation is whether the errors occur during minus strand or plus strand synthesis. Two observations that favor a predominantly minus strand error mechanism are (i) the known lower fidelity of reverse transcriptase when using an RNA template [43], and (ii) the complete absence of base mismatches detected during DNA sequencing (for instance, an original G/U base mismatch should yield Sanger strand reads consisting of a mixture of $\mathrm{G} / \mathrm{C}$ and $\mathrm{A} / \mathrm{T}$ at that site after PCR amplification). The occurrence of predominantly minus strand errors is suggested because a mismatch generated during (-) strand synthesis would always be removed during degradation of the plus strand RNA by HIV RNaseH, whereas mismatches occurring during plus strand synthesis would remain. Although DNA repair could repair plus strand mismatches, the low repair capacity of nondividing cells makes this unlikely compared to the simple minus strand mechanism. Consistent with this idea, a recent deep sequencing study concluded that minus strand deamination events catalyzed by A3G were only inefficiently repaired by UBER or mismatch repair in T cells, which have much greater repair activity than MDM [44].

\section{Conclusions}

These in vitro results inform on the previous detection of dUMP in both alveolar macrophages and peripheral blood monocytes of HIV patients on ART [4]. We find that the kinetics for MC infection is 
compatible with their lifetime in vivo and their near absence of UBER activity is consistent with the retention of viral dUMP at high levels at least until differentiation into macrophages, where UBER becomes possible. Thus, the fate of uracilated proviruses would appear to center on the expression levels of UBER enzymes in different cellular environments and whether infected macrophages can enter the repair competent $\mathrm{G}_{1}$ state. A full understanding of these complexities in the context of in vivo derived macrophages is therefore essential. Our current understanding suggests that potential therapeutic opportunities may exist for eradicating densely uracilated proviruses if UBER could be activated under conditions where subsequent repair is interrupted pharmacologically.

\section{Methods}

Cells. Monocytes (MC) were purified from peripheral blood mononuclear cells (PBMC) of HIV negative consenting donors under Johns Hopkins University IRB approval (IRB00038590) using a Ficoll-Hypaque density gradient followed by negative selection using a Pan monocyte isolation kit (Miltenyi Biotech). Monocyte purified by the pan monocyte isolation kit were checked for T-cell contamination by RT-PCR using TCR- $\beta$ primer pairs which gave $99 \%$ purity (see detail in Supplemental Methods). In some cases, to prevent cell adherence and differentiation into monocyte-derived macrophages (MDM), MC were cultured up to 7 days in suspension using ultra-low adherence 96-well plates (Corning) using RPMI 1640 (Gibco) supplemented with $10 \%$ donor autologous serum, $100 \mu \mathrm{g} / \mathrm{mL}$ penicillin, $100 \mu \mathrm{g} / \mathrm{mL}$ streptomycin (HyClone), $0.3 \mathrm{mg} / \mathrm{ml}$ glutamine and $10 \mathrm{mM}$ HEPES (RPMI-AS). Fully-differentiated MDM were generated by culturing MC under adherent conditions for seven days using RPMI-AS medium supplemented with 10 $\mathrm{ng} / \mathrm{mL}$ M-CSF (R\&D Systems). T cells were purified from PBMC using T cell pan isolation kit (Miltenyi Biotec). T cells were first cultured for three days using stimulating conditions (RPMI supplemented with $10 \%$ (vol/vol) donor autologous serum, $100 \mu \mathrm{g} / \mathrm{mL}$ penicillin, $100 \mu \mathrm{g} / \mathrm{mL}$ streptomycin (HyClone), 0.3 $\mathrm{mg} / \mathrm{ml}$ glutamine $1 \mathrm{mg} / \mathrm{ml}$ of Phytohemagglutinin (Gibco) and, ciprofloxacin ( $5 \mathrm{mg} / \mathrm{ml})$ ). T cells were then infected with $\mathrm{HIV}^{\mathrm{BaL}}$ and cultured for up to 7 days in RPMI medium additionally supplemented with $10 \mathrm{U} / \mathrm{ml}$ recombinant interleukin-2 (Sigma). The dividing cell lines HEK293T (ATCC) and HAP1 (Horizon Discovery) were seeded at 2 million cells per T-75 flask. The culture media for the dividing cells ( $\mathrm{HI}-10)$ consisted of RPMI-1640 medium $+10 \%$ heat inactivated FBS (Sigma), $100 \mu \mathrm{g} / \mathrm{mL}$ penicillin and 100 $\mu \mathrm{g} / \mathrm{mL}$ streptomycin. Cells were grown until $70 \%$ confluence with media changes every $48 \mathrm{~h}$. The cells were released from the flask using $5 \mathrm{~mL}$ of $0.05 \%$ trypsin-EDTA (Gibco), followed by washing three times with PBS (Gibco) without $\mathrm{CaCl}_{2}$ or $\mathrm{MgCl}_{2}$.

Single nucleotide polymerase extension assay. The extraction of total dNTPs from 1-2 million cells and quantification of dUTP and dTTP were performed as previously described [3], except that HIV-1 reverse transcriptase (RTase) was used in the extension assay (Millipore-Sigma). Further details are described in the Supplemental Methods.

Western Blotting. Extracts for western blotting were prepared using denaturing conditions with approximately 4 million MC or 2 million MDM that were collected from culture plates by treatment with trypsin. Further details are described in the Supplemental Methods. 
Uracil DNA glycosylase activity assay. The hUNG activity present in cell extracts was measured using a molecular beacon fluorescent hairpin DNA substrate containing U/A base pairs as previously described $[3,4]$. The UNG activity was normalized to the total extract protein. The measured uracil excision activity was fully inhibited by the addition of uracil DNA glycosylase inhibitor protein (UGI) to the extracts, establishing that only hUNG and not any other glycosylase activity was being detected.

Activity of HIV reverse transcriptase with dUTP and dTTP substrates. To test whether dUMP is an efficient substrate for reverse transcription by HIV RTase, we measured its capacity to distinguish between dTTP and dUTP incorporation using the SNE assay described above. For these measurements we assembled two $100 \mu \mathrm{L}$ reactions with dNTP mixtures consisting of $1200 \mathrm{fmol}$ dCTP, dGTP, dATP and containing either dTTP or dUTP. The reactions contained $600 \mathrm{fmol}$ of a 5'-FAM-labeled DNA templateprimer with a single A overhang on the template strand. To these reactions $1 \mu \mathrm{L}$ (2 units) of recombinant HIV RTase was added and incubated at $37^{\circ} \mathrm{C}$. Ten $\mu \mathrm{L}$ samples were removed at $0,10,20,30,60,120,240$, $2400 \mathrm{~s}$, quenched in $40 \mu \mathrm{L}$ of $98 \%$ formamide containing $20 \mathrm{mM}$ EDTA (pH 8.0), and resolved on a $15 \%$ denaturing polyacrylamide gel. The initial linear rates of dUTP or dTTP incorporation into the primer strand were measured by fitting data points at $<40 \%$ reaction.

Viruses and infections. The macrophage tropic replicative HIV- 1 virus HXB3/BaL was obtained from the NIH AIDS Reagent Program (Catalog \#11414) and propagated in MOLT-4/CCR5 cells. Culture supernatants of infected MOLT-4/CCR5 cells were collected at 12 to $15 \mathrm{dpi}$, centrifuged to clear cellular debris, passed through $0.45 \mu \mathrm{m}$ filters, aliquoted, and stored at $-80^{\circ} \mathrm{C}$. Vesicular stomatitis virus $\mathrm{G}$ protein (VSV-G) pseudo-typed HIV-1 virions (HIVNL4.3 $3^{(\mathrm{VSVG})}$ ) were generated as previously described (3) by cotransfection of HEK 293T cells with pNL4-3- $\triangle E$-eGFP and pVSV-G. pCW57.1.FL.UNG lentiviral particles (LV) were generated by transfecting HEK 293 T cells with; $30 \mu \mathrm{g}$ of pCW $57.1 \mathrm{hUNG}, 30 \mu \mathrm{g}$ of pMDLg/pRRE packaging plasmid (containing Gag \& Pol, Addgene), $12 \mu \mathrm{g}$ of pRSV-Rev packaging plasmid (containing Rev, Addgene), $6 \mu \mathrm{g}$ of pMD2.g packaging plasmid (containing VSV-G envelope, Addgene). Transfection was performed using Lipofectamin 2000 (Invitrogen) following the manufacturers protocol. Lentivral particles were then concentrated over $20 \%$ sucrose cushion and ultracentrifugation at $28 \mathrm{k} \mathrm{RPM}$ for two hours. Viral titer was determined by the ELISA p24 antigen assay (Lenti-X p24 Rapid Titer Kit, TaKaRa). Monocytes were infected immediately after purification by adding HIV- ${ }^{\text {BaL }}$ virus to the culture at $\mathrm{MOI}$ between 3 and 5 based on the p24 titer. After infection, monocytes were cultured in two different manners: (i) non-adherent growth conditions in the absence of M-CSF to minimize differentiation by adherence or cytokine mechanisms or, (ii) by adherent growth for seven days in the presence of M-CSF to promote differentiation into MDM over the infection. Infections of fully differentiated MDM were performed after adherent growth in the presence of M-CSF for seven days. Just prior to addition of virus to MDM, the media was replaced with RPMI $+10 \% \mathrm{dFCS}$ and omitting M-CSF. Monocytes and MDM were also cultured in the presence of $5 \mathrm{mM}$ thymidine (Sigma) overnight to increase the intracellular dTTP levels prior to infection with HIV-1 ${ }^{\mathrm{BaL}}$ ("thymidine rescue" conditions).

Viral copy number using qPCR and ddPCR. Viral copy numbers were determined using qPCR. A standard curve for viral copy number quantification was developed using 10-fold serial dilutions of DNA extracted 
from J-lat cells that contain a single integrated HIV per genome (NIH AIDS Reagent Program). Real time qPCR was performed using a Qiagen Rotor-Gene qPCR instrument using the Rotor-gene Probe PCR kit. Reactions were performed in a $25 \mu \mathrm{l}$ reaction volume using $0.4 \mathrm{iM}$ forward and reverse primers and 0.2 $\mu \mathrm{M}$ of specific hydrolysis probes. PCR targeted the early or late HIV reverse transcripts (ERT or LRT). Amplification was performed using a two-step program: initial heating at $95^{\circ} \mathrm{C}$ for five minutes, followed by 40 cycles of denaturation at $95^{\circ} \mathrm{C}$ for 10 seconds and annealing and extension at $60{ }^{\circ} \mathrm{C}$ for 30 seconds. For determination of viral copies per cell, simultaneous quantification of the genomic RnaseP (RPP30) gene was performed using published primers and a specific probe as previously described [4]. All primers and probes used in this study are listed in Supplemental Table 1.

Determination of HIV proviral DNA using alu-gag nested PCR. To determine copy numbers of HIV proviral DNA, a nested PCR method was used [26]. The first PCR was performed using a forward primer that targeted genomic alu sequences randomly located near integrated proviruses $(0.2 \mu \mathrm{M})$ and an HIVspecific gag reverse primer $(1.2 \mu \mathrm{M})$. Other PCR conditions were $200 \mu \mathrm{M}$ of each dNTP, 1X LongAmp taq buffer (NEB), 5 units of LongAmp taq DNA polymerase (NEB) and $50 \mathrm{ng}$ of DNA sample extracted from infected cells in a $50 \mu \mathrm{l}$ final reaction volume. Amplification was performed using the following thermocycler program: initial activation heating $94^{\circ} \mathrm{C}$ for two min, followed by 20 cycles of denaturation at $94{ }^{\circ} \mathrm{C}$ for $30 \mathrm{sec}$, annealing at $50^{\circ} \mathrm{C}$ for $30 \mathrm{sec}$ and extension at $65^{\circ} \mathrm{C}$ for three min and a final extension reaction at $65{ }^{\circ} \mathrm{C}$ for ten minutes. The PCR product is diluted 20 -fold and $5 \mu$ l of the diluted PCR product is used as an input material for the second PCR reaction, which is performed using LRT forward and reverse primers and probe using the Rotor Gene Probe PCR kit (Qiagen) as described above. Proviral copy numbers were determined using the J-lat cell integration standard as described above. Genomic copy numbers were determined using the RPP30 qPCR measurement described above using the same amount of input total DNA sample used to measure proviral copy numbers.

Uracil content of viral DNA. Uracil content of viral DNA was determined using either uracil excision droplet digital PCR (Ex-ddPCR) or a similar qPCR method (Ex-qPCR) as previously described [4] with some modifications as described in the Supplemental Methods.

RT-qPCR of extracellular viral RNA. Culture supernatants were collected from infected $M C, M^{\mathrm{EI}}$ or $M M^{L l}$ 7-days post infection unless otherwise specified. Supernatants were first spun to remove cellular debris, filtered using $0.22 \mu \mathrm{m}$ filter and frozen at $-80^{\circ} \mathrm{C}$ until use. RNA was extracted from $140 \mu$ of culture supernatant using the Qiagen mini-Viral Prep kit according to the manufacturer's protocol. RNA $(\sim 3 \mu \mathrm{g})$ was treated with 2 units turbo-DNase (Invitrogen) in 1X turbo-DNase buffer for 30 min at $37^{\circ} \mathrm{C}$, to remove DNA carryover and then purified using Qiagen RNEasy kit. About $1.3 \mu \mathrm{g}$ of RNA was used to generated cDNA with the Qiagen OmniScript cDNA preparation kit following the manufacturers protocol. Using the cDNA as input material and the Rotor Gene qPCR probe kit (Qiagen), the genomic HIV RNA copies in the supernatants of infected MC and MDM cultures were measured relative to a standard curve developed with the J-lat HIV integration standard cell line (see above). Thermal cycling conditions for GPCR 
consisted of $95^{\circ} \mathrm{C}$ for $5 \mathrm{~min}$, and 40 cycles of denaturation at $95^{\circ} \mathrm{C}$ for $10 \mathrm{sec}$ and annealing and extension at $60^{\circ} \mathrm{C}$ for $30 \mathrm{sec}$. The measurements are reported as RNA copies per provirus present.

Sequencing of single viral reverse transcripts and genomic RNA copies. Monocytes and MDMs were infected with HIV-1 ${ }^{\mathrm{BaL}}$ as described above and culture extracts and supernatants were collected at 7 days post infection, filtered and frozen at $-80^{\circ} \mathrm{C}$ until use. A combination of QPCR and limiting-dilution PCR were performed to obtain samples that contained single copies of viral DNA and RNA as determined by the fraction of qPCR-positive samples at each dilution. Dilutions where one out of five replicates tested positive were taken as clonal according to Poisson statistics [4,9]. Amplified single copies were then sequenced by the Sanger method. Further details are described in the Supplemental Methods.

Infectivity of virus isolated from MC and MDM. MOLT-4/CCR5 target cells were infected overnight at an MOI of 0.5 (0.1 pg p24/cell) using HIV-1 ${ }^{\text {BaL }}$ virus collected from three different producer culture supernatants: (i) MC infected immediately after isolation and cultured for 7 days under non-adherent conditions in the absence of M-CSF to maintain a monocyte-like phenotype, (ii) infected monocytes that were cultured for seven days in the presence of M-CSF [we refer to these cells as "early infection" macrophages $\left(\mathrm{MDM}^{\mathrm{EI}}\right)$ to indicate that they were infected before differentiating into MDM], and (iii) MDM that were infected after differentiating for 7 days under adherent conditions in the presence of M-CSF [we refer to these infected cells as $\mathrm{MDM}^{\mathrm{L}}$ ) to indicate that infection occurred after the macrophage phenotype was achieved]. One day after the addition of the virus to the MOLT-4/CCR5 cells, the cells were washed and cultured in RPMI 1640 (Invitrogen) supplemented with 10\% heat inactivated bovine serum, $100 \mathrm{U} / \mathrm{ml}$ penicillin, $100 \mu \mathrm{g} / \mathrm{ml}$ streptomycin, $0.3 \mathrm{mg} / \mathrm{ml}$ glutamine and $200 \mu \mathrm{g} / \mathrm{ml}$ neomycin. DNA was extracted at 7 days post infection and proviral DNA levels were measured by alu-gag qPCR. Viral protein 24 (p24) levels in the culture supernatants were measured at day 12 using ELISA.

Flow Cytometry. Flow cytometric measurements were made on freshly isolated MC or MDM obtained by differentiation for 7 days in the presence of M-CSF. Prior to flow cytometry, adherent MDM were washed twice with Hank's balanced salt solution (HBSS) and released from the culture flask by treating with Accutase (Cell Technologies) for $30 \mathrm{~min}$ at $37^{\circ} \mathrm{C}$. MC and MDM were resuspended at a concentration of $10^{6} \mathrm{cells} / \mathrm{mL}$ in $1 \times$ HBSS (pH 7.2), $5 \mathrm{mM}$ EDTA and $0.5 \%$ BSA and passed through a $35 \mu \mathrm{m}$ nylon mesh (BD Biosciences) before injection on a FACSCalibur flow cytometer (Becton Dickinson). For each cell type, 20,000 events were recorded for analysis. Dot plots were created using forward scatter (FSC-H) and side scatter (SSC-H) measurements to determine the size and granularity differences. All data were analyzed using Cell Quest software.

Light Microscopy. MC were prepared as described above using the Pan-Monocyte isolation kit from Miltenyi. The obtained MC were resuspended in fresh RPMI medium and allowed to adhere on a glass slide for an hour before collecting images at 20x magnification. MDM were differentiated from MCs as described above using RPMI media containing 10\% autologous sera and M-CSF. After seven days, the adherent cells were visualized using 20x magnification and images were collected. Images were converted to 8-bit grayscale using ImageJ software. 
Statistics. The data uncertainties are expressed as the mean \pm 1 SD. Unless otherwise indicated in the figure legends, data are derived from at least three independent experiments using cells isolated from at least three donors. Experiments using cells from individual donors were performed in triplicate. Data were analyzed using the Prism 8.0 statistical program from GraphPad Software. Pairwise statistical analysis of test groups was performed by a two-tailed unpaired Student's $t$-test, assuming equal variances between groups. The significance of the mutation frequencies observed between two groups was determined using a chi-squared analysis.

\section{List Of Abbreviations}

APE1: apurinic/apyrimidinic endonuclease 1

APOBEC: apolipoprotein B mRNA editing enzyme, catalytic polypeptide-like

ART: Antiretroviral therapy

ddPCR: droplet digital polymerase chain reaction

dNTP: deoxy nucleotide triphosphate

dTMP: deoxy thymidine monophosphate

dTTP: deoxy thymidine triphosphate

dUMP: deoxy uridine diphosphate

dUTP: deoxy uridine triphosphate

ERT: Early reverse transcriptase

Ex-qPCR: Excision quantitative polymerase chain reaction

HIV: Human Immunodeficiency Virus

hUNG: human uracil-N glycosylase

LRT: Late reverse transcriptase

MC: Monocyte

M-CSF: Macrophage colony stimulating factor

MDM: Monocyte derived macrophages

$\mathrm{MDM}^{\mathrm{El}}$ : Monocyte derived macrophage early infected 
$\mathrm{MDM}^{\mathrm{LI}}$ : Monocyte derived macrophage late infected

MOI: Multiplicity of infection

RTase: Reverse transcriptase

SAMHD1: sterile a motif and HD domain-containing protein 1

UBER: Uracil base excision repair

\section{Declarations}

\section{Ethics approval and consent to participate}

The human subject protocols and ethics were reviewed and approved by the Johns Hopkins Institutional Review Board (IRB approval IRB00038590)

\section{Consent for publication}

Not Applicable

\section{Acknowledgments}

The authors acknowledge the support of the Johns Hopkins Institute for Clinical and Translational Research team for recruiting, consenting and performing blood draws from healthy subjects.

\section{Author contributions}

JS conceived and designed the experiments and wrote the paper. MM performed or was involved in the design and execution of all experiments and contributed to manuscript preparation and writing, $A E$ performed immunoblotting experiments, UNG and RTase activity measurements and contributed to figure preparation and writing, JC performed RT-qPCR measurements and figure preparation, NC performed nucleotide pool measurements, and SS generated viral constructs and performed viral infectivity measurements. All authors read and approved the final manuscript.

\section{Competing interests}

The authors declare that they have no competing interests.

\section{Funding}

This work was supported by NIH grants R01 Al124777 (J.T.S.), R01-GM056834 (J.T.S.) and an AmFAR Innovation Grant 108834-55-RGRL (J.T.S.). A. E. was supported by an American Heart fellowship 19POST34380400. 
Availability of data and material

The datasets used and/or analyzed during the current study are available from the corresponding author on reasonable request.

\section{References}

1. Gandhi SK, Siliciano JD, Bailey JR, Siliciano RF, Blankson JN. Role of APOBEC3G/F-Mediated Hypermutation in the Control of Human Immunodeficiency Virus Type 1 in Elite Suppressors. J Virol. 2008;82:3125-30.

2. Harris RS, Dudley JP. APOBECs and virus restriction. Virology. 2015;479-480:131-45.

3. Weil AF, Ghosh D, Zhou Y, Seiple L, McMahon MA, Spivak AM, et al. Uracil DNA glycosylase initiates degradation of HIV-1 cDNA containing misincorporated dUTP and prevents viral integration. Proc Natl Acad Sci U S A. 2013;110:E448-57.

4. Hansen EC, Ransom M, Hesselberth JR, Hosmane NN, Capoferri AA, Bruner KM, et al. Diverse fates of uracilated HIV-1 DNA during infection of myeloid lineage cells. Elife [Internet]. 2016;5. Available from: http://dx.doi.org/10.7554/eLife.18447

5. Priet S, Gros N, Navarro J-M, Boretto J, Canard B, Quérat G, et al. HIV-1-associated uracil DNA glycosylase activity controls dUTP misincorporation in viral DNA and is essential to the HIV-1 life cycle. Mol Cell. 2005;17:479-90.

6. Yan N, O'Day E, Wheeler LA, Engelman A, Lieberman J. HIV DNA is heavily uracilated, which protects it from autointegration. Proc Natl Acad Sci U S A. 2011;108:9244-9.

7. Vértessy BG, Tóth J. Keeping uracil out of DNA: physiological role, structure and catalytic mechanism of dUTPases. Acc Chem Res. 2009;42:97-106.

8. Priet S, Sire J, Quérat G. Uracils as a cellular weapon against viruses and mechanisms of viral escape. Curr HIV Res. 2006;4:31-42.

9. Cui J, Gizzi A, Stivers JT. Deoxyuridine in DNA has an inhibitory and promutagenic effect on RNA transcription by diverse RNA polymerases. Nucleic Acids Res. 2019;47:4153-68.

10. Zhang H, Yang B, Pomerantz RJ, Zhang C, Arunachalam SC, Gao L. The cytidine deaminase CEM15 induces hypermutation in newly synthesized HIV-1 DNA. Nature. 2003;424:94-8.

11. Amie SM, Noble E, Kim B. Intracellular nucleotide levels and the control of retroviral infections. Virology. 2013;436:247-54.

12. Gavegnano C, Kennedy EM, Kim B, Schinazi RF. The Impact of Macrophage Nucleotide Pools on HIV-1 Reverse Transcription, Viral Replication, and the Development of Novel Antiviral Agents. Mol Biol Int. 2012;2012:625983.

13. Eldin P, Chazal N, Fenard D, Bernard E, Guichou J-F, Briant L. Vpr expression abolishes the capacity of HIV-1 infected cells to repair uracilated DNA. Nucleic Acids Res. 2014;42:1698-710.

14. Ahn J, Vu T, Novince Z, Guerrero-Santoro J, Rapic-Otrin V, Gronenborn AM. HIV-1 Vpr loads uracil DNA glycosylase-2 onto DCAF1, a substrate recognition subunit of a cullin 4A-ring E3 ubiquitin ligase for proteasome-dependent degradation. J Biol Chem. 2010;285:37333-41.

15. Schröfelbauer B, Yu Q, Zeitlin SG, Landau NR. Human immunodeficiency virus type 1 Vpr induces the 
degradation of the UNG and SMUG uracil-DNA glycosylases. J Virol. 2005;79:10978-87.

16. Langevin C, Maidou-Peindara P, Aas PA, Jacquot G, Otterlei M, Slupphaug G, et al. Human immunodeficiency virus type $1 \mathrm{Vpr}$ modulates cellular expression of UNG2 via a negative transcriptional effect. J Virol. 2009;83:10256-63.

17. Rogstad DK, Liu P, Burdzy A, Lin SS, Sowers LC. Endogenous DNA lesions can inhibit the binding of the AP-1 (c-Jun) transcription factor. Biochemistry. 2002;41:8093-102.

18. Sonza S, Mutimer HP, Oelrichs R, Jardine D, Harvey K, Dunne A, et al. Monocytes harbour replicationcompetent, non-latent HIV-1 in patients on highly active antiretroviral therapy. AIDS. 2001;15:17-22.

19. Calcaterra S, Cappiello G, Di Caro A, Garbuglia AR, Benedetto A. Comparative analysis of total and integrated HIV-1 DNA in peripheral CD4 lymphocytes and monocytes after long treatment with HAART. J Infect. 2001;43:239-45.

20. Ziegler-Heitbrock L. Reprint of: Monocyte subsets in man and other species. Cell Immunol. 2014;291:11-5.

21. Crowe S, Zhu T, Muller WA. The contribution of monocyte infection and trafficking to viral persistence, and maintenance of the viral reservoir in HIV infection. 2017;74:635-41.

22. Nottet HS, Persidsky Y, Sasseville VG, Nukuna AN, Bock P, Zhai QH, et al. Mechanisms for the transendothelial migration of HIV-1-infected monocytes into brain. J Immunol. 1996;156:1284-95.

23. Persidsky Y, Stins M, Way D, Witte MH, Weinand M, Kim KS, et al. A model for monocyte migration through the blood-brain barrier during HIV-1 encephalitis. J Immunol. 1997;158:3499-510.

24. Kennedy EM, Daddacha W, Slater R, Gavegnano C, Fromentin E, Schinazi RF, et al. Abundant noncanonical dUTP found in primary human macrophages drives its frequent incorporation by HIV-1 reverse transcriptase. J Biol Chem. 2011;286:25047-55.

25. Bauer M, Goldstein M, Christmann M, Becker H, Heylmann D, Kaina B. Human monocytes are severely impaired in base and DNA double-strand break repair that renders them vulnerable to oxidative stress. Proceedings of the National Academy of Sciences. 2011;108:21105-10.

26. O’Doherty U, Swiggard WJ, Jeyakumar D, McGain D, Malim MH. A sensitive, quantitative assay for human immunodeficiency virus type 1 integration. J Virol. 2002;76:10942-50.

27. Mlcochova P, Sutherland KA, Watters SA, Bertoli C, de Bruin RAM, Rehwinkel J, et al. A G1-like state allows HIV-1 to bypass SAMHD1 restriction in macrophages. EMBO J. John Wiley \& Sons, Ltd; 2017;36:604-16.

28. Carr CE, Khutsishvili I, Gold B, Marky LA. Thermodynamic Stability of DNA Duplexes Comprising the Simplest T $\rightarrow$ dU Substitutions. Biochemistry. 2018;57:5666-71.

29. Parker JB, Stivers JT. Dynamics of uracil and 5-fluorouracil in DNA. Biochemistry. 2011;50:612-7. 30. Lichtenstein DL, Rushlow KE, Cook RF, Raabe ML, Swardson CJ, Kociba GJ, et al. Replication in vitro and in vivo of an equine infectious anemia virus mutant deficient in dUTPase activity. J Virol. 1995;69:2881-8.

31. Turelli P, Pétursson G, Guiguen F, Mornex JF, Vigne R, Quérat G. Replication properties of dUTPasedeficient mutants of caprine and ovine lentiviruses. J Virol. 1996;70:1213-7.

32. Threadgill DS, Steagall WK, Flaherty MT, Fuller FJ, Perry ST, Rushlow KE, et al. Characterization of equine infectious anemia virus dUTPase: growth properties of a dUTPase-deficient mutant. J Virol. 
1993;67:2592-600.

33. Steagall WK, Robek MD, Perry ST, Fuller FJ, Payne SL. Incorporation of uracil into viral DNA correlates with reduced replication of EIAV in macrophages. Virology. 1995;210:302-13.

34. Mansky LM, Preveral S, Selig L, Benarous R, Benichou S. The interaction of vpr with uracil DNA glycosylase modulates the human immunodeficiency virus type 1 In vivo mutation rate. J Virol. 2000;74:7039-47.

35. Guenzel CA, Hérate C, Le Rouzic E, Maidou-Peindara P, Sadler HA, Rouyez M-C, et al. Recruitment of the nuclear form of uracil DNA glycosylase into virus particles participates in the full infectivity of HIV-1. J Virol. 2012;86:2533-44.

36. Meyerhans A, Vartanian JP, Hultgren C, Plikat U, Karlsson A, Wang L, et al. Restriction and enhancement of human immunodeficiency virus type 1 replication by modulation of intracellular deoxynucleoside triphosphate pools. J Virol. 1994;68:535-40.

37. Diamond TL, Roshal M, Jamburuthugoda VK, Reynolds HM, Merriam AR, Lee KY, et al. Macrophage tropism of HIV-1 depends on efficient cellular dNTP utilization by reverse transcriptase. J Biol Chem. 2004;279:51545-53.

38. Brown A, Zhang H, Lopez P, Pardo CA, Gartner S. In vitro modeling of the HIV-macrophage reservoir. J Leukoc Biol. 2006;80:1127-35.

39. Cromer D, Schlub TE, Smyth RP, Grimm AJ, Chopra A, Mallal S, et al. HIV-1 Mutation and Recombination Rates Are Different in Macrophages and T-cells. Viruses. 2016;8:118. 40. Curlin ME, Zioni R, Hawes SE, Liu Y, Deng W, Gottlieb GS, et al. HIV-1 envelope subregion length variation during disease progression. PLoS Pathog. 2010;6:e1001228.

41. Zolla-Pazner S, Cardozo T. Structure-function relationships of HIV-1 envelope sequence-variable regions refocus vaccine design. Nat Rev Immunol. 2010;10:527-35.

42. Wärmländer S, Sponer JE, Sponer J, Leijon M. The Influence of the Thymine C5 Methyl Group on Spontaneous Base Pair Breathing in DNA. J Biol Chem. 2002;277:28491-7.

43. Abram ME, Ferris AL, Shao W, Alvord WG, Hughes SH. Nature, Position, and Frequency of Mutations Made in a Single Cycle of HIV-1 Replication. J Virol. 2010;84:9864-78.

44. Pollpeter D, Parsons M, Sobala AE, Coxhead S, Lang RD, Bruns AM, et al. Deep sequencing of HIV-1 reverse transcripts reveals the multifaceted antiviral functions of APOBEC3G. Nat Microbiol. 2018;3:22033.

\section{Figures}




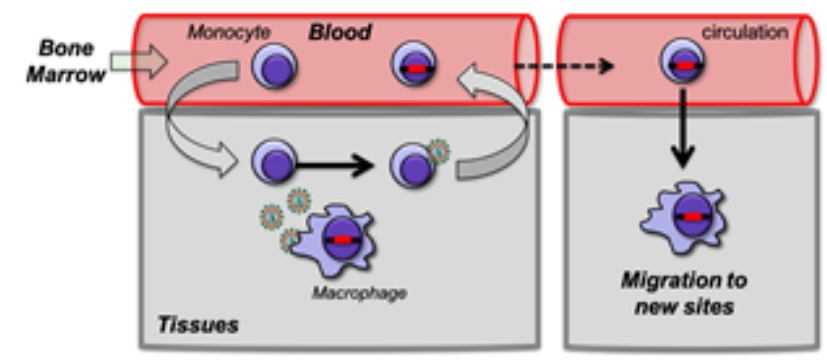

Figure 1

Model for HIV infection of tissue macrophages and infection of new sites via migration of infected monocytes. The model depicts two potential pathways by which macrophages become infected with HIV based on their state of differentiation at the time of virus encounter. In the first scenario, fully differentiated tissue macrophages are infected by contact with virus arising from infected cells passing through the tissue. Alternatively, circulating bone marrow derived monocytes, which are the precursors to some tissue macrophages, could be infected prior to their differentiation into macrophages, either through contact with infected macrophages or T cells. This pathway may be relevant for in vivo HIV infection because proviruses have been detected in circulating monocytes of HIV infected individuals who are on ART and have undetectable levels of serum virus.

a

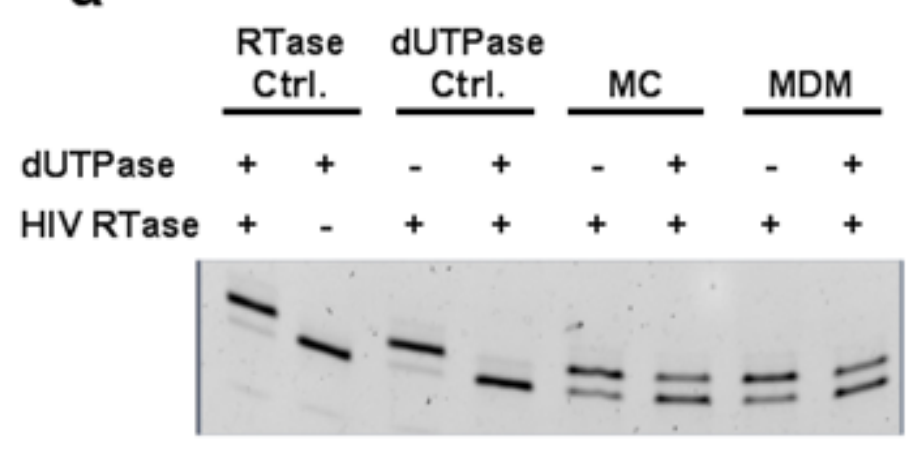

C

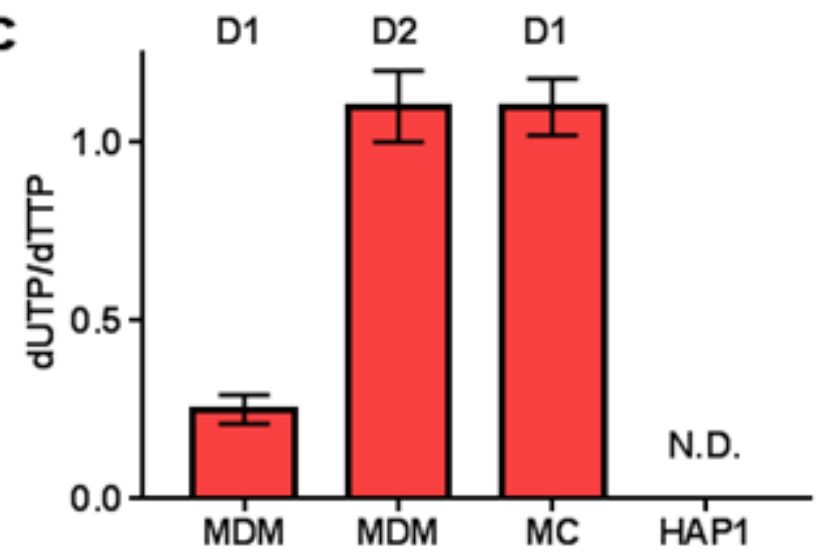

b

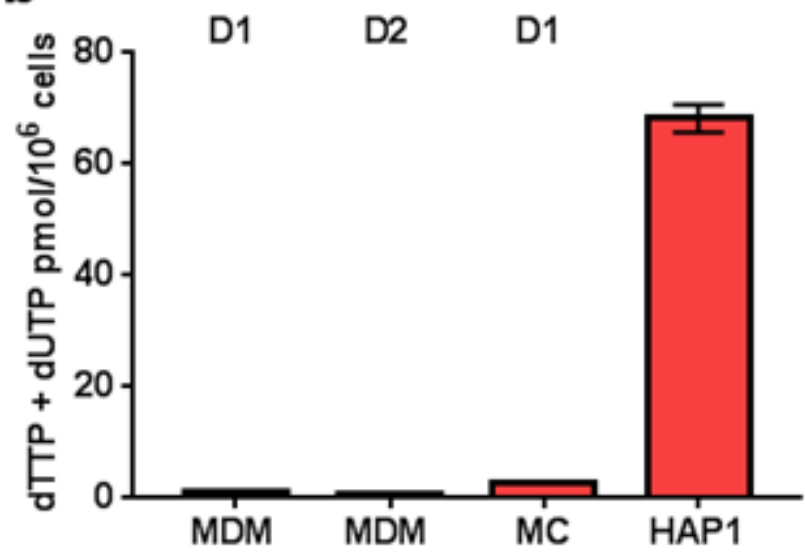

d

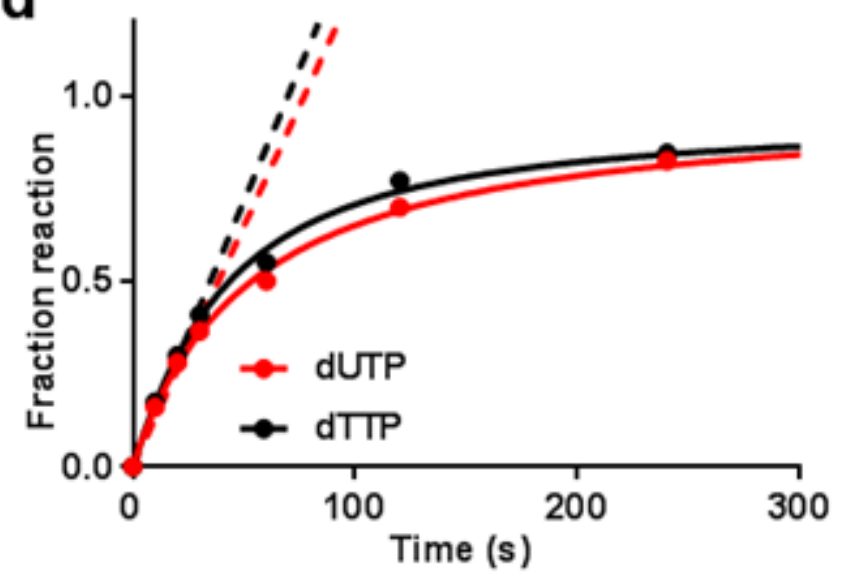

Figure 2 
dUTP and dTTP pool measurements in extracts from MDM, MC and comparison with Hap1 dividing cells. A single nucleotide extension assay (SNE) was used to measure dUTP and dTTP levels in MDM, MC and the Hap1 dividing cell line (Fig. S1). The specific measurement of dUTP in a mixed pool of TTP + dUTP is accomplished by the in vitro conversion of dUTP dUMP + PPi using dUTPase prior to the SNE assay. (a) Typical SNE assay to determine dUTP levels in cell dNTP extracts. The RTase control (dTTP only) establishes that RTase is required for converting the $n$ bp substrate into $n+1$ and that dUTPase does not inhibit extension in the absence of dUTP (see methods). The dUTPase control establishes that RTase can fully extend the probe by a single nucleotide in the presence of a dNTP mixture with dUTP replacing dTTP. The addition of dUTPase completely abolishes extension under these conditions. (b) Combined dTTP + dUTP pool measurements for MDM and MC from two donors (D1 and D2) and the HAP1 dividing cell line. Error bars are standard errors from triplicate measurements. (c) dUTP/dTTP ratio for the various cell types. ND, not detected. Error bars are standard errors from triplicate measurements. (d) Determination of the relative activity of HIV RTase for incorporation of dTTP or dUTP opposite to adenine. The solid line is a theoretical fit to the entire time course, while the dashed lines are initial rate linear fits, which are statistically indistinguishable. The concentration of the template DNA was $50 \mathrm{nM}$ and concentration of each dNTP was $100 \mathrm{nM}$, which is the estimated concentration of these dNTPs in MDM or MC. 

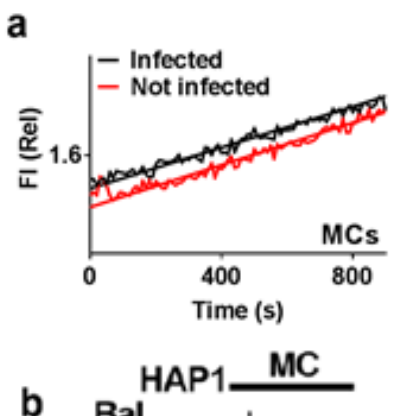

b $\mathrm{Bal}-+$

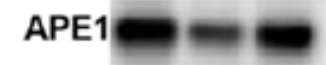

Tub

C

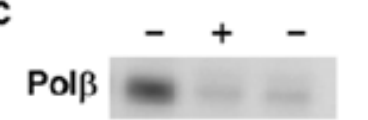

Tub
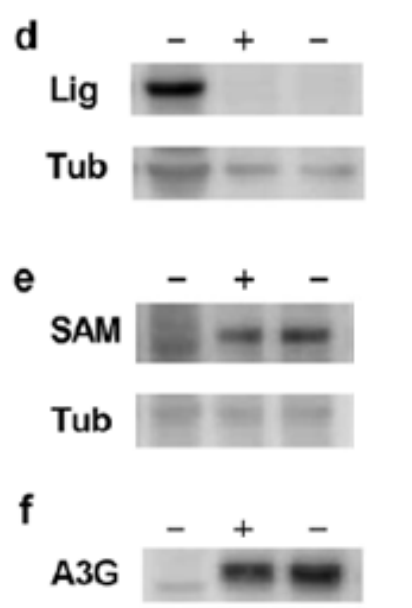

A3A

Tub
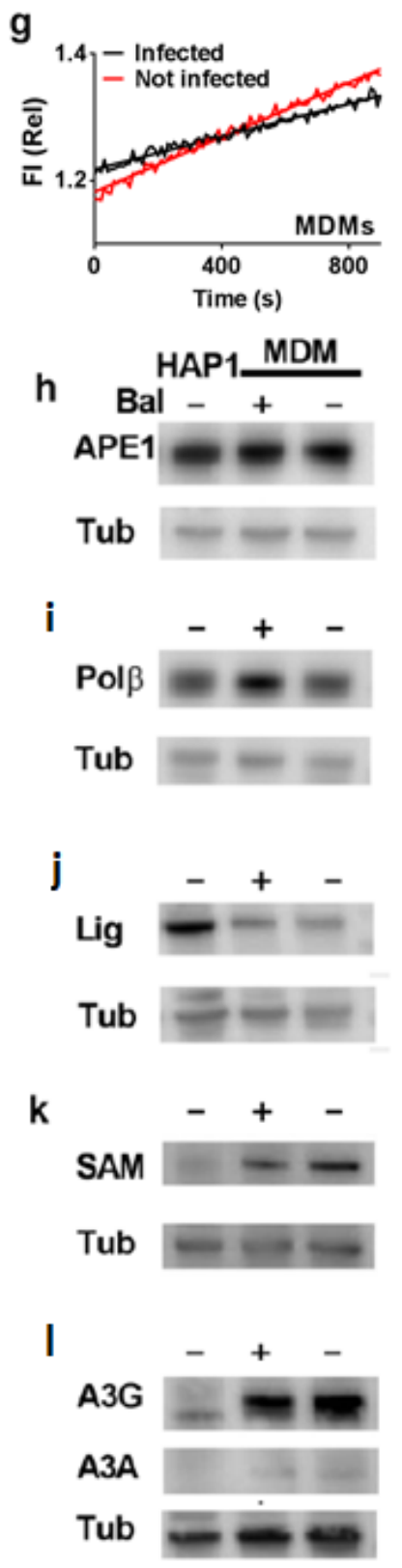

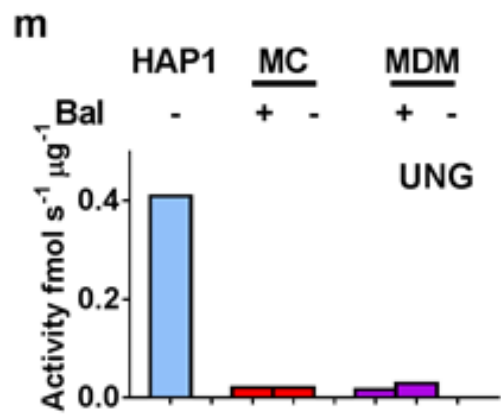

$q$

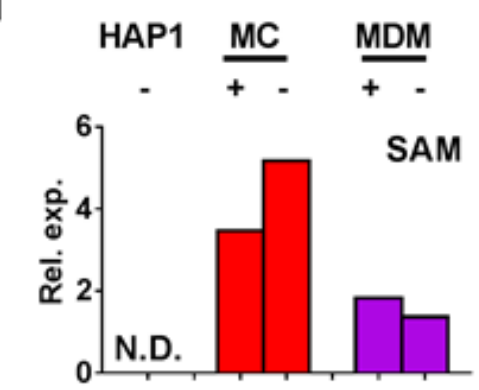

n

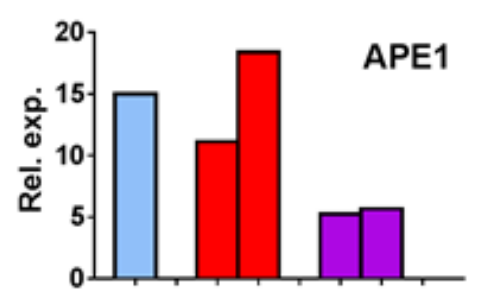

o
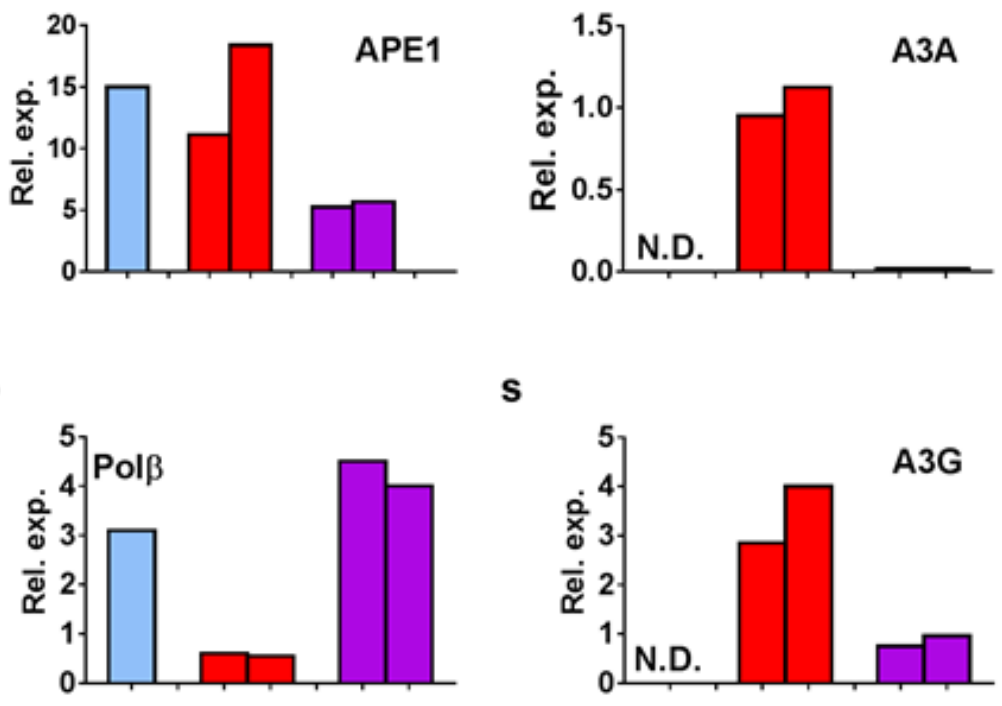

S

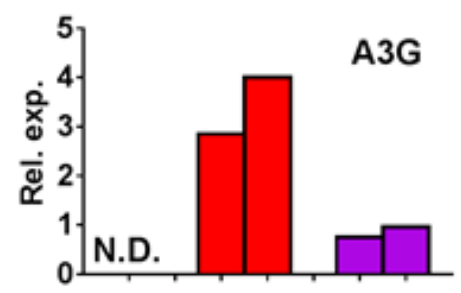

\section{Figure 3}

p

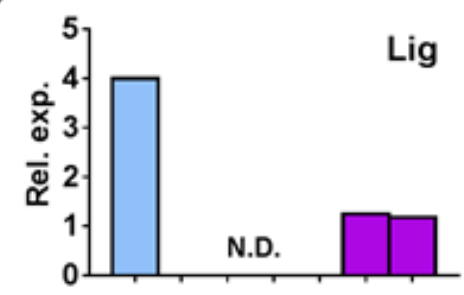

Determination of uracil base excitation repair (UBER) capacity of infected and uninfected MCs, MDMs and comparison with the HAP1 dividing cell line. MC or MDM were infected with replication competent CCR5 tropic HIV-1BAL virus at MOI = 5. Extracts from MC (2nd lane in panels b, c, d, e, f) and MDM (3rd lane in panels $h, I, j, k, I)$ using uninfected $(-)$ or infected cells $(+)$ at 3 days post-infection were processed for western blotting. Blots were performed using $10 \mu \mathrm{g}$ of total cell protein, except for Lig Illa, where $20 \mu \mathrm{g}$ was used. Due to the low levels of hUNG present in non-dividing cells, native extracts $(10 \mu \mathrm{g})$ were prepared for measurement of UNG enzymatic activity using a sensitive real-time fluorescence assay (see methods). The specific measurement of UNG activity was established by the addition of UNG inhibitor UGI $(4 \mu \mathrm{M})$, which completely inhibited the measured activity. Panels a to i show western blots or activity assays for detection of $(\mathrm{a}, \mathrm{g})$ uracil DNA glycosylase (UNG); (b, h) AP endonuclease 1, (APE1); DNA 
polymerase $\beta$, (pol $\beta)(c, i)$; (d, j) ligase III (Lig); (e, k) SAMHD1 (SAM); and (f, I). APOBECA3A (A3A) and APOBECA3G (A3G); tubulin (Tub) loading control was used in each blot. Panels $m$ to s show the relative expression level (Rel. exp.) or activity of each enzyme for each cell line as indicated in each panel.

Relative expression was calculated by dividing the band intensity of the protein of interest by the intensity of loading control after correction for background.



Figure 4 
Characteristics of HIV infection in MC and macrophages infected before differentiation (MDMEI) and after differentiation (MDMLI). (a) Infection of MC and MDM with HIV-1BaL virus was performed using three conditions $(\mathrm{MOI}=5)$. In one condition, $\mathrm{MC}$ were immediately infected and then cultured using nonadherent conditions without M-CSF for seven days to maintain a monocyte phenotype. MDMEl refers to "early infection" of freshly isolated MC, which were subsequently differentiated into MDM under adherent conditions in the presence of M-CSF. MDMLI refers to "late infection" where MDM were first fully differentiated before infection. In all cases, HIV fusion inhibitor drug, enfuvirtide, (10 $\mu \mathrm{M}$ final concentration) was added to culture medium after 24 hours of infection to prevent multiple rounds of infection. (b) Fraction of provirus in MC, MDMEl and MDMLI that contain uracil as determined by alu-gag Ex-qPCR. ND: not detected (c) Time course for appearance of early reverse transcription (ERT) products as determined by RT-qPCR using a primer set targeting the 5'LTR region of the HIV genome. (d) Time course for appearance of late reverse transcription (LRT) products as determined by RT-qPCR using a primer set targeting the LTR region of the HIV genome. (e) Time course for appearance of proviral DNA copies determined by alu-gag GPCR. Identical infections activated T cells were used as controls and are shown in panels d, e and f. Abbrev: ND, not detected. * not done. All data are averages obtained from three blood donors. 

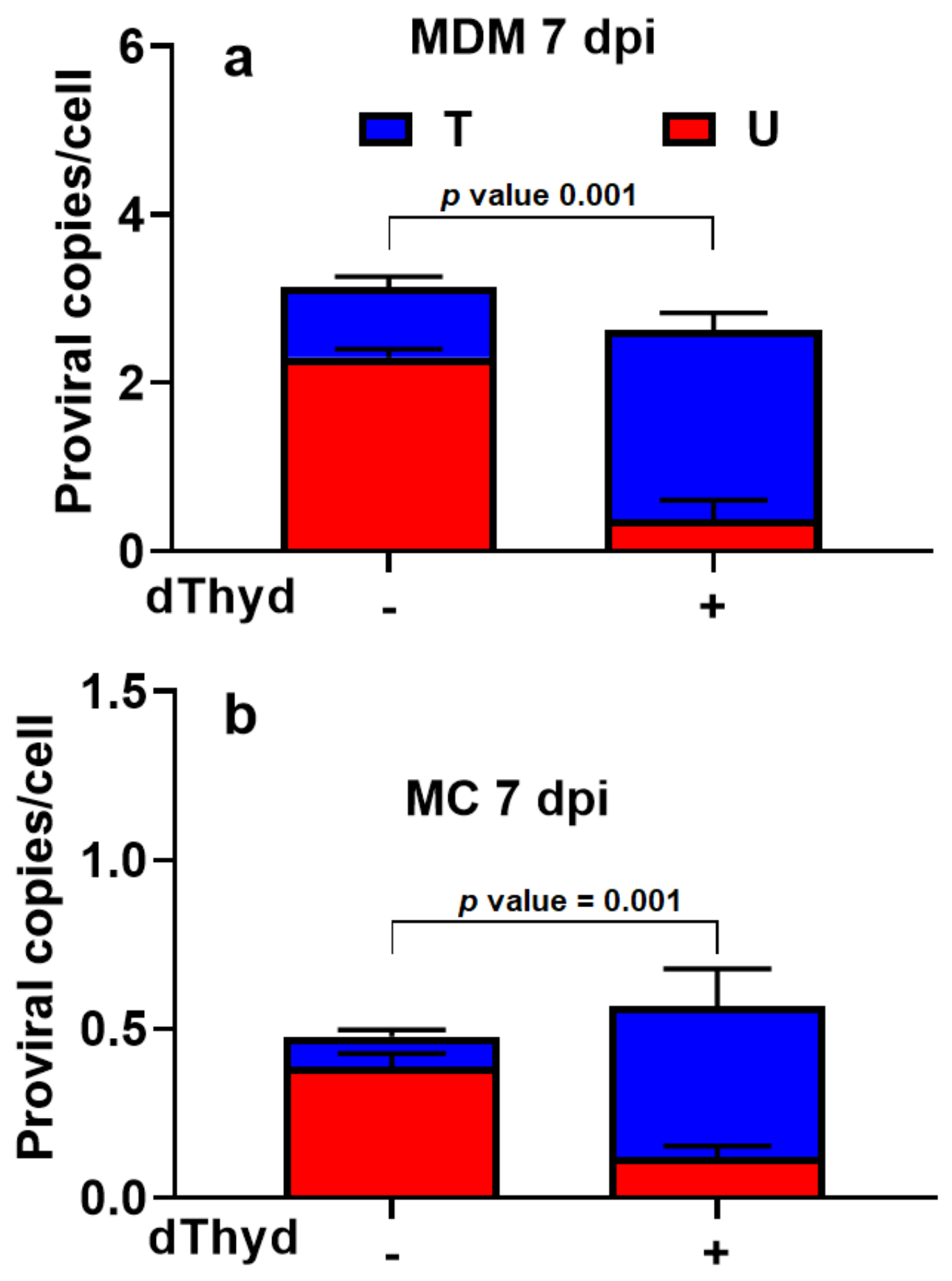

Figure 5

dUMP in HIV DNA during infection of MC and MDM arises predominantly from dUTP. MC and MDM were infected with HIV-1Bal virus at an MOI of 5 (1 pg p24/cell) in the presence (+) and absence (-) of $5 \mathrm{mM}$ deoxythymidine (dThyd). Copy number measurements of uracilated (red) and non-uracilated (blue) proviruses were made 7 dpi using the Alu-gag Ex-qPCR method. (a) MDM and (b) MC infected in the absence and presence of dThyd. p-values from an unpaired Student's t-test are shown. 

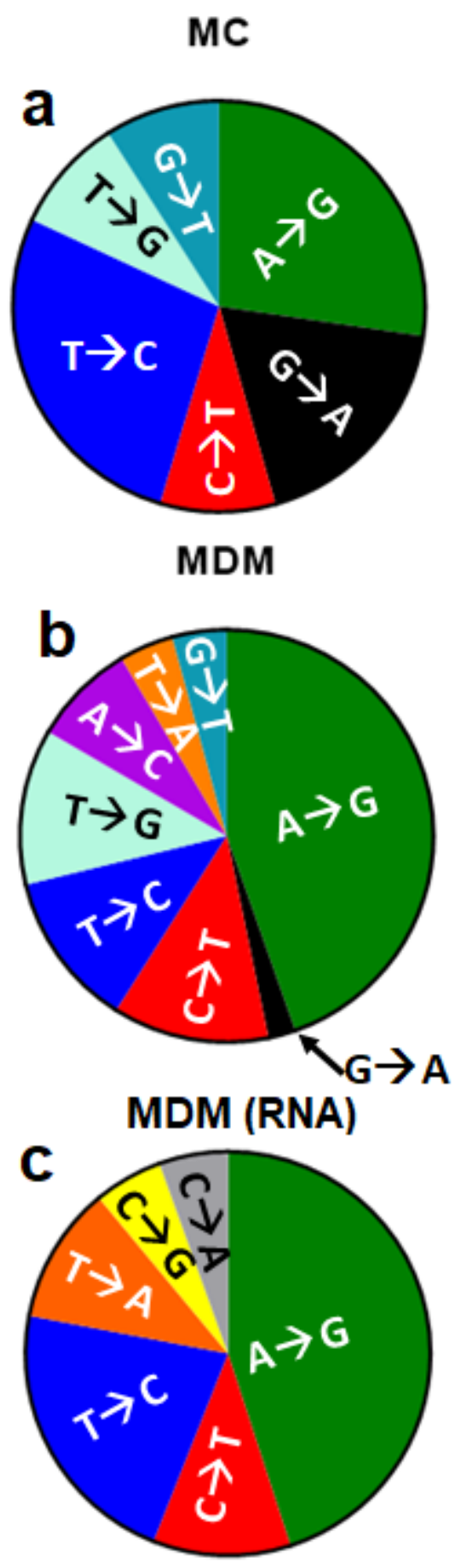

\section{Figure 6}

Mutagenic effects of uracil incorporation on HIV proviral DNA and extracellular viral RNA in MDM and MC. Sequences of single proviral clones and extracellular viral RNA from infected MDM and MC. MC and MDMs were infected with equivalent amount of virus (1 pg p24/cell). Seven days after infection total cellular DNA and extracellular viral RNA were extracted from cells and the culture supernatants, respectively. Single viral copies were amplified by limiting-dilution nested PCR and sequenced by the Sanger method. Sequences were aligned to our laboratory reference HIV-1BaL sequence. (a) Mutational spectrum of HIV proviral DNA sequences from infected MC. (b) Mutational spectrum of HIV proviral DNA 
sequences from infected MDM (c) Mutational spectrum of extracellular viral RNA extracted from infected MDM.

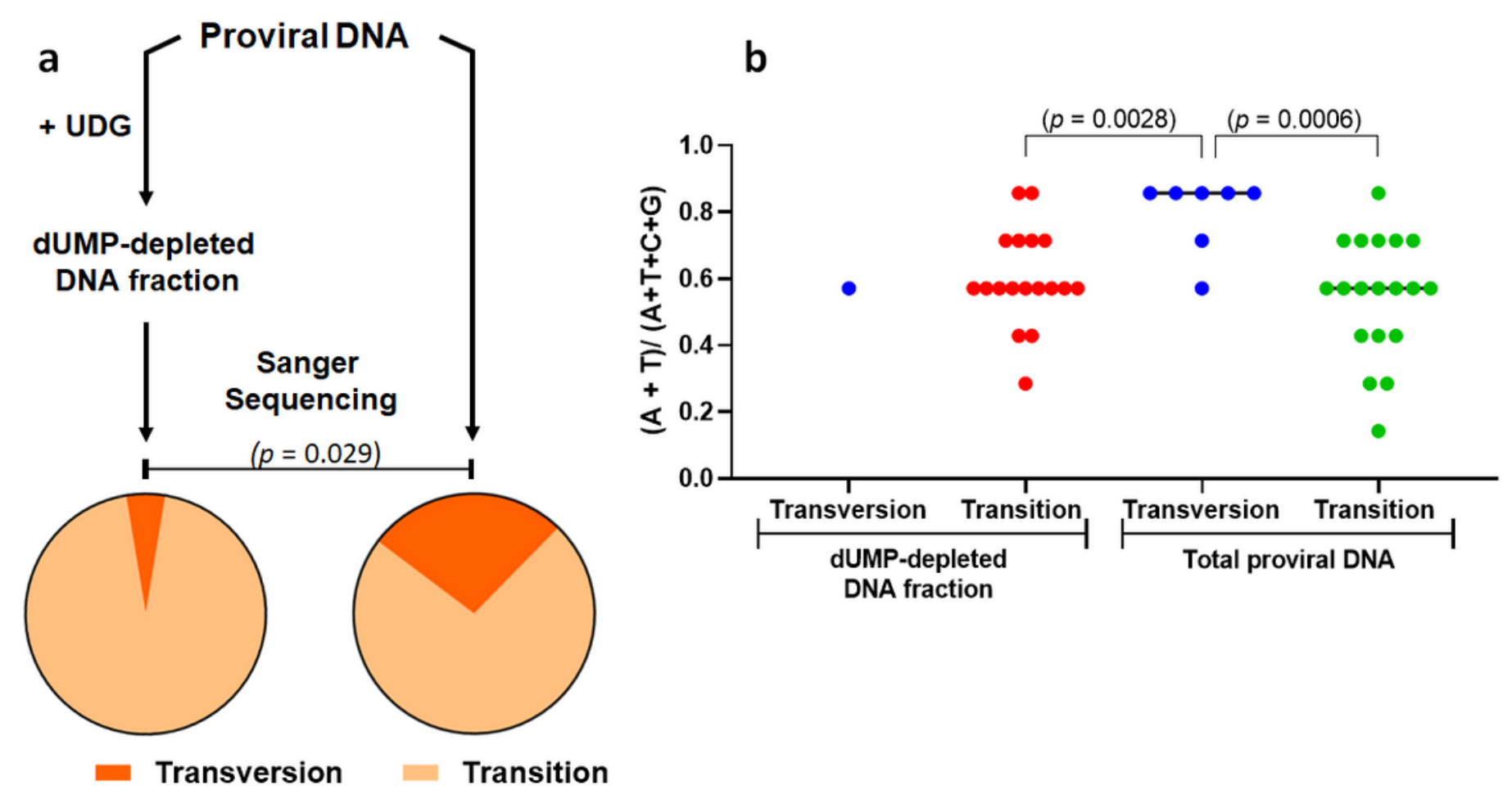

Figure 7

Viral cDNA containing dUMP contains unique $(+)$ strand transversion mutations that correlate with $A / T(U)$ content surrounding the transversion site. MDMs were infected (1 pg p24/cell). Seven days after infection total cellular DNA was extracted from cells. Single copy sequences were determined by limiting-dilution nested PCR and Sanger sequencing. Sequences were aligned to our laboratory reference HIVBaL sequence. (a) A population of viral cDNAs was sequenced before and after the removal of dUMPcontaining sequences as indicated. Removal of dUMP-containing viral sequences was accomplished by treatment of the total DNA population with UNG prior to PCR amplification and clonal sequencing. HIV proviral DNA sequences where the dUMP fraction was subtracted contained almost exclusively transition mutations (left pie chart), while the total proviral DNA without subtraction showed the same transition mutations but also unique transversion mutations (right pie chart). (b) Transversion mutations are associated with loci that contain higher average $A / T(U)$ content. In this analysis, the average $A+T(U)$ content over a window of three flanking bases on both sides of each transversion or transition mutation was calculated. The loci with transversion mutations had a higher average content than the 29 loci that showed transition mutations. p-values from an unpaired Student's t-test indicate the statistical significance of the average $A+T(U)$ content in the six base window surrounding the transition and transversion sites. 

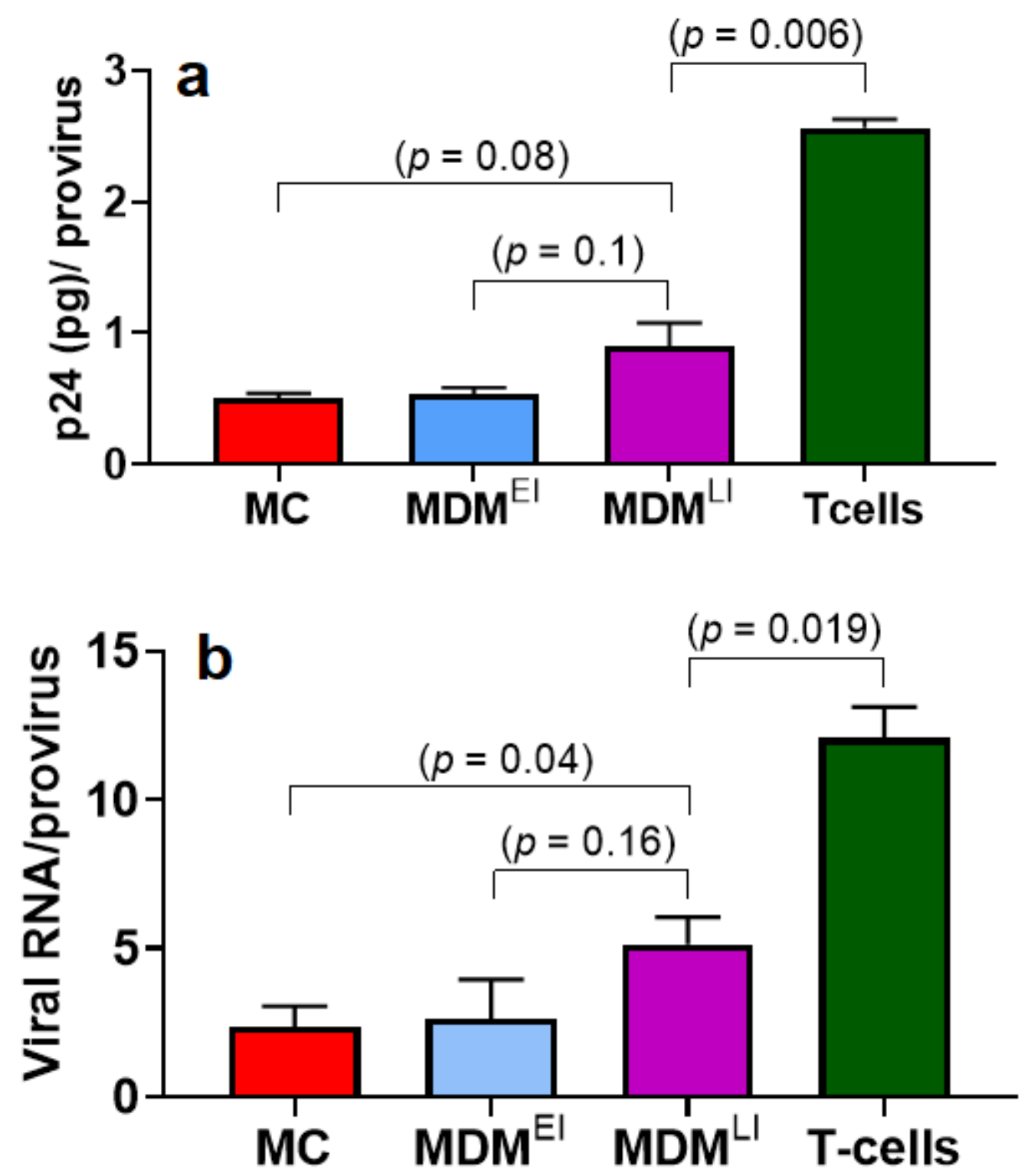

Figure 8

Efficiency of viral particle production from HIV proviruses in MC, MDM and activated T cells. All cell types were infected with Bal virus at $\mathrm{MOI}$ of 5 (1 pg p24/cell) and seven days after infection culture supernatants and cellular DNA were processed. MC were infected immediately after isolation and then cultured for seven days without differentiation using nonadherent conditions in the absence of M-CSF. MDMEI were infected as freshly isolated MC and then differentiated into MDM under adherent conditions in the presence of M-CSF. MDMLI were infected after differentiation for seven days in the presence of MCSF. T cell controls were infected and cultured in IL-2 medium for seven days. T20 entry inhibitor $(10 \mu \mathrm{M})$ was used to prevent reinfection. (a) Viral protein 24 (p24) levels in culture supernatants were measured 
by ELISA and normalized to total proviral copies determined by alu-gag qPCR. (b) Extracellular viral RNA was measured using RT-PCR and normalized to total proviral copies determined by alu-gag qPCR. pvalues from an unpaired Student's t-test are shown.
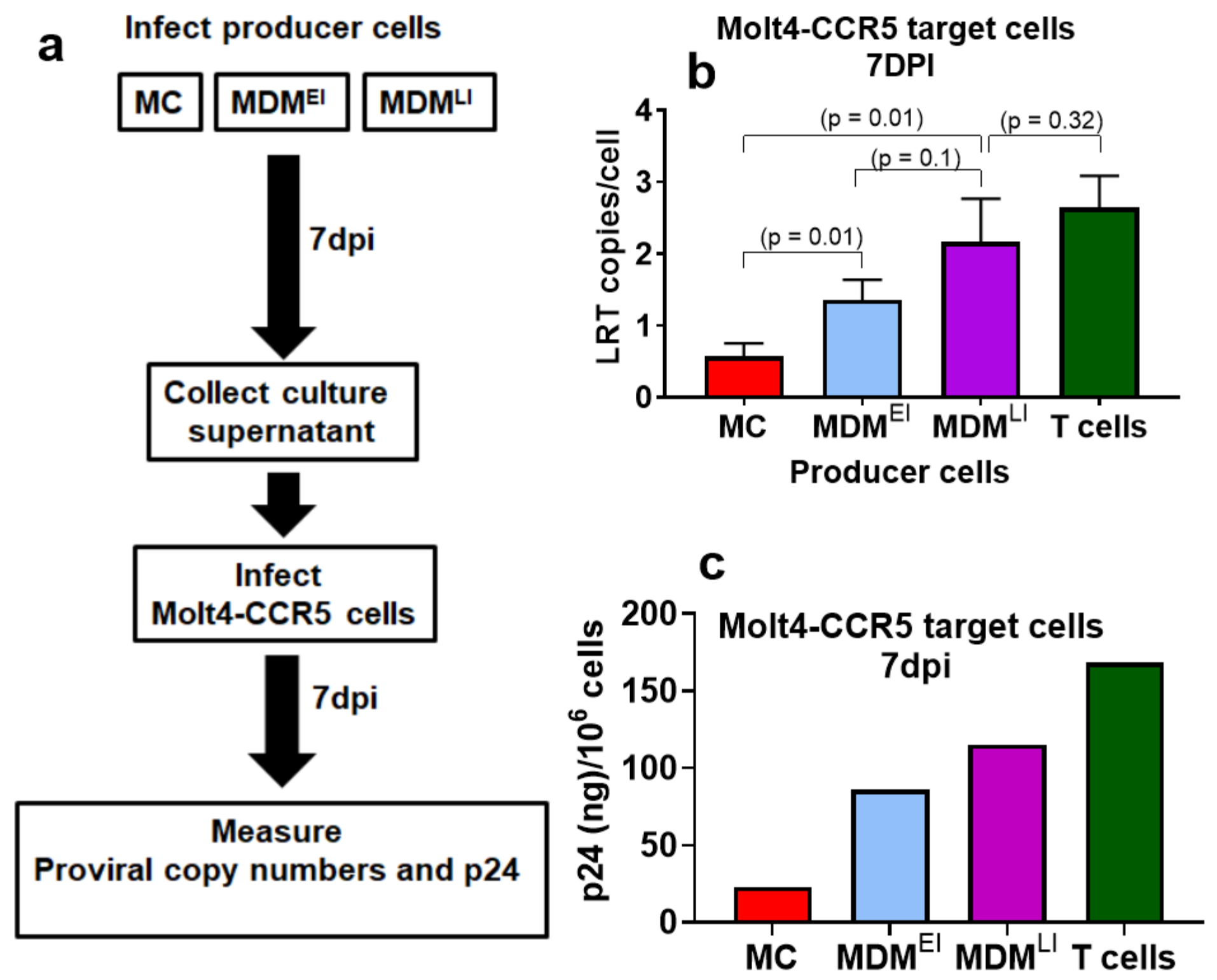

Figure 9

Efficiency of viral particle production from HIV proviruses in MC, MDM and activated T cells. (a) Experimental scheme for determining infectivity of virus generated from MC, MDMEl and MDMLI producer cells. (b) Viral supernatants from MC, MDMEI and MDMLI and T cell producer cell cultures were collected at $7 \mathrm{dpi}$ and MOLT-4/CCR5 target cells were infected using a $0.1 \mathrm{pg}$ p24/target cell. Seven days after infection of target cells, cellular DNA was extracted and levels of proviral DNA were determined by qPCR. Relative infectivity is shown as LRT copies per target cell. (c) p24 levels as measured by ELISA (single donor). p-values from an unpaired Student's t-test are shown. 
a hUNG inducecd before HIV ${ }^{N L 4-3}$ infection
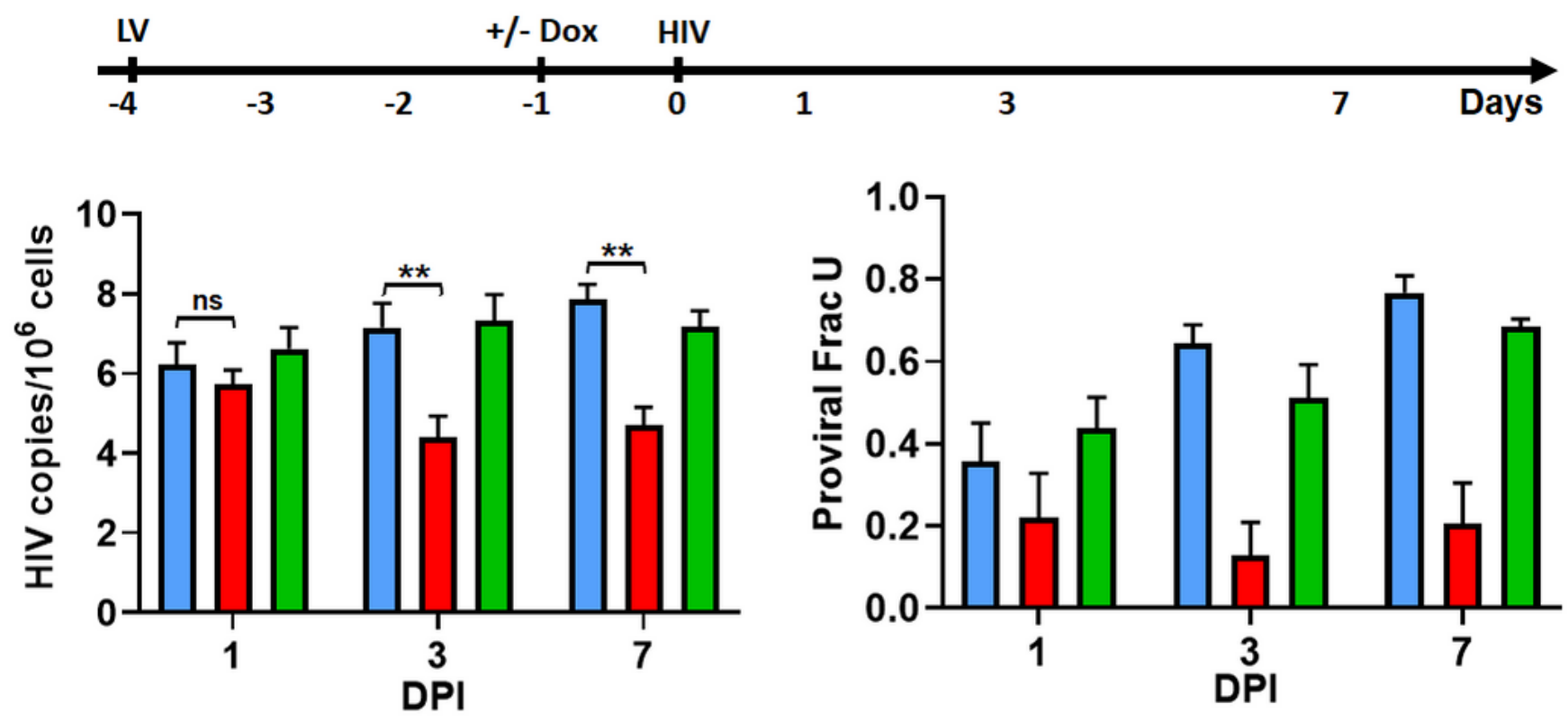

b hUNG induced after HIV ${ }^{\text {NL4-3 infection }}$

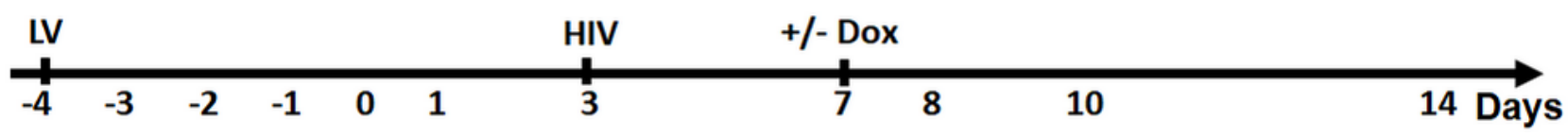

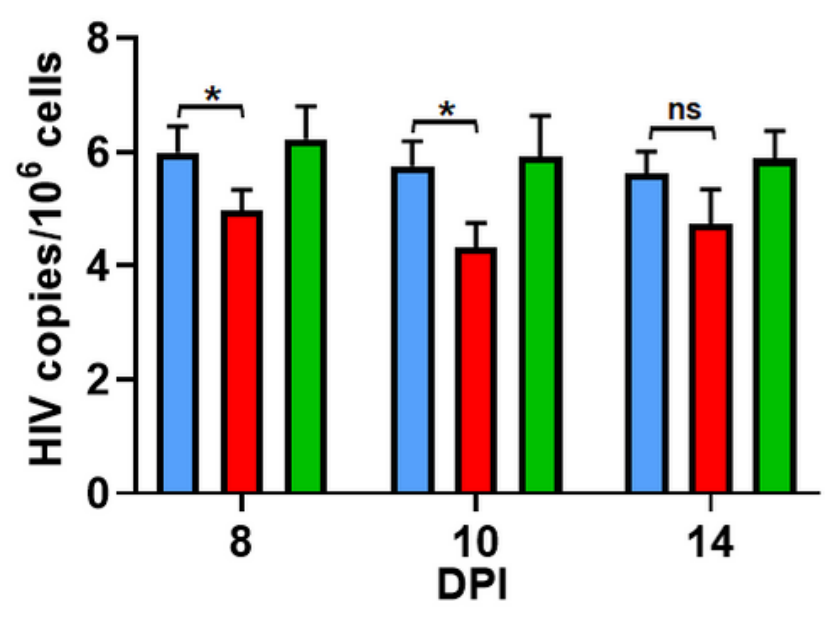

$\square$ Uninduced

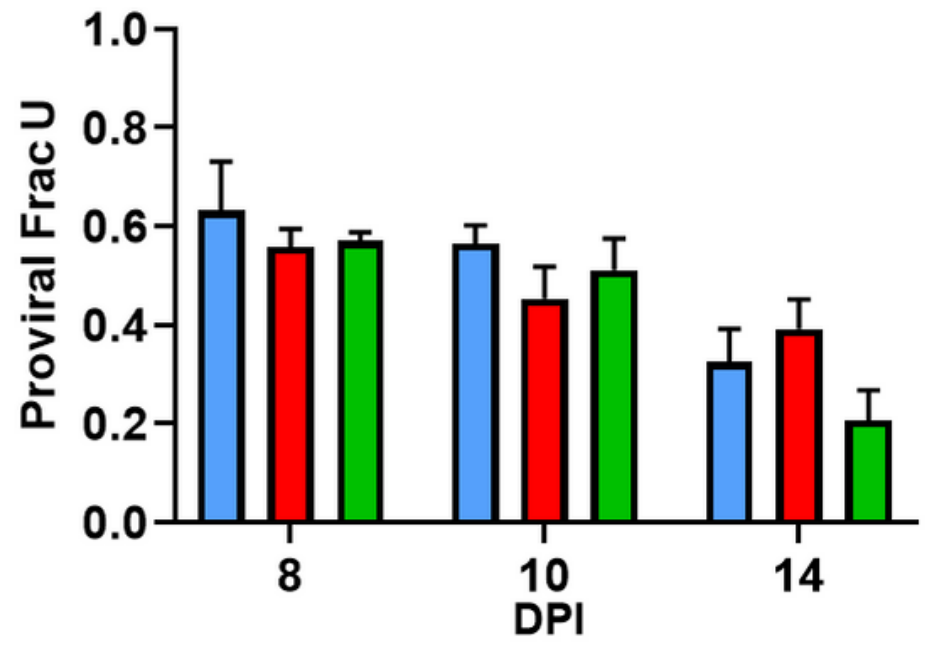

Induced $\square$ HIV $^{\text {NL4-3 }}$

Figure 10

Exogenous expression of hUNG2 depletes uracilated HIV DNA products. Exogenous full-length hUNG2 was over expressed in MDM under control of a doxycycline (Dox) inducible promoter by lentiviral transduction $(\mathrm{MOI}=5)$. The timelines above each panel indicate the experimental course and the points where hUNG2 expression was induced relative to infection with HIVNL4-3. (a) hUNG2 expression was induced with doxycycline $(1 \mu \mathrm{g} / \mathrm{ml})$ one day before HIVNL4-3 infection. Proviral copy numbers (left panel) 
and the fraction of HIV proviral DNA that contained dUMP were measured at indicated times (FracU, right panel). (b) Expression of hUNG2 was induced 7-days after infection with HIVNL4-3 in order to allow HIV to fully integrate. Proviral copy numbers (left panel) and the fraction of HIV proviral DNA that contained dUMP were measured at indicated times (FracU, right panel). Uninduced (blue) and no lentiviral infection (green) controls were also performed.

\section{Supplementary Files}

This is a list of supplementary files associated with this preprint. Click to download.

- 12302019SupplementalMethodsandFigures.docx 